SJ Quinney College of Law, University of Utah Utah Law Digital Commons

4-2021

Blockchain Initiatives for Tax Administration

Young Ran Kim

Follow this and additional works at: https://dc.law.utah.edu/scholarship

Part of the Tax Law Commons 


\title{
BLOCKCHAIN INITIATIVES FOR TAX ADMINISTRATION
}

\author{
69 UCLA LAW REV. (forthcoming)
}

\section{Young Ran (Christine) Kim *}

A thriving body of literature discusses various legal issues related to blockchain, but often it mixes the discussion about blockchain with cryptocurrency. However, blockchain is not the same as cryptocurrency. Defined as a decentralized, immutable, peer-to-leer ledger technology, blockchain is a newly emerging data management system. The private sector-including the financial industry and supply chains - and the public sector-property records, public health, voting, and compliance, have all begun to utilize blockchain. Since more data is processed remotely, and thus digitally, the evolution of blockchain is gaining stronger momentum.

While scholarship on blockchain is growing, none of the scholarship has considered the impact of blockchain on the tax sector. This Article extends the study of blockchain to tax administration, evaluates the feasibility of incorporating blockchain within existing tax administrations, and provides policymakers with criteria to consider and some recommended designs for blockchain. Blockchain can enhance the efficiency and transparency of tax administration through its ability to deliver reliable, real-time information from many sources to a large audience. Further, a well-designed private consortium blockchain, evolved from the classic public blockchain, may effectively protect taxpayers' information. Potential areas that blockchain could enhance are payroll taxes, withholding taxes, value added taxes, transfer pricing, the sharing of information between federal, state, and local governments as well as countries.

This Article offers normative considerations for policymakers deliberating blockchain initiatives for tax administration, such as timeline, standardization, its integration with other systems, its limitations, and the

\footnotetext{
*Associate Professor of Law, University of Utah S.J. Quinney College of Law. For helpful comments, I am grateful to Michael Berwind (IRS Office of Chief Counsel), Yariv Brauner, Bryan Call (IRS Office of Chief Counsel), Liz Chien (Ripple), Jorge Contreras, Mindy Herzfeld, Ariel Jurow Kleiman, Sarah Lawsky, Leandra Lederman, Roberta Mann, Omri Marian, Erin Scharff, Lawrence Zlatkin (CoinBase), participants of the National Tax Association Annual Conference, University of Florida International Tax Symposium, University of Oregon Tax Policy Colloquium, University of Utah Faculty Workshop, Law and Society Association Annual Meeting, Rocky Mountain Junior Scholars Forum, and SEALS Annual Conference. Special thanks to the University of Utah's Albert and Elaine Borchard Fund for Faculty Excellence for its generous support. Scott Allen, Casey Bond, Simeon Brown, Shad Edwards, Eli LeCates, Leland Stanford McCullough, Brooks Lindberg, and Jessica Ramirez provided excellent research assistance.
} 
accompanying legislation to regulate the government and the taxpayer's rights and privacy. Those implications may resonate with a broader audience beyond tax policymakers.

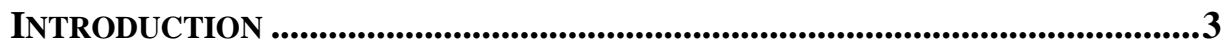

I. UNPACKING BLOCKCHAIN............................................................10

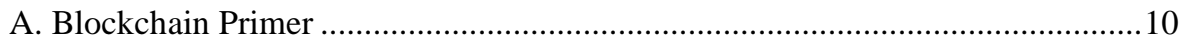

1. A Distributed, Immutable, Peer-to-Peer Ledger........................................... 10

2. Blockchain's Key Features, Pros, and Cons .............................................15

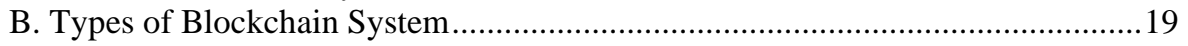

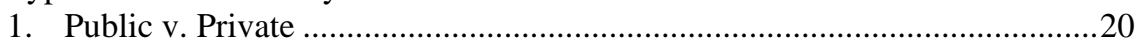

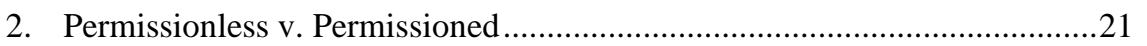

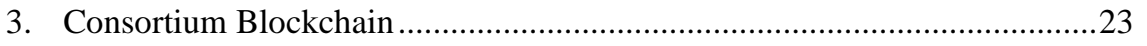

C. Applied Blockchains in the Private and Public Sectors ......................................24

1. Private Sector Applications ....................................................................24

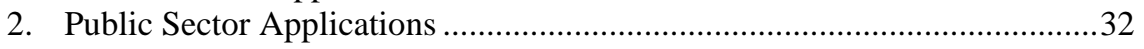

II. BLOCKCHAIN ARCHITECTURE FOR TAX ADMINISTRATION....................39

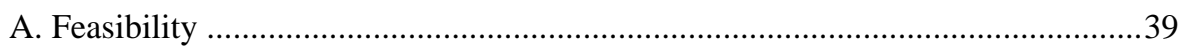

B. Recommending a Private Consortium Blockchain...............................................41

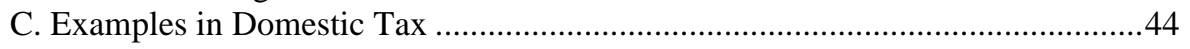

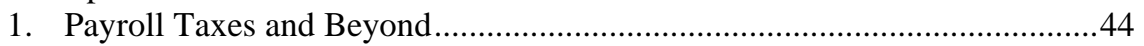

2. Value Added Taxes ......................................................................................46

3. Information Sharing among Federal, State and Local Governments.............48

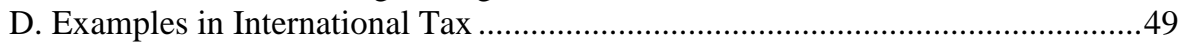

1. Transfer Pricing, Country-by-County Reporting ..........................................50

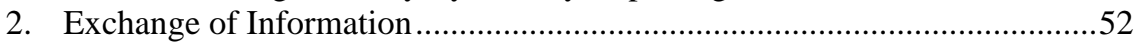

III. NORMATIVE CONSIDERATIONS FOR BLOCKCHAIN IN TAX....................54

A. When is a Good Time to Incorporate Blockchain? ..............................................54

B. Standardization and Integration .....................................................................57

C. At the Intersection Between Offline and Digital: Limitations of Reducing Tax Gap...

D. Vili's Governance Paradox and the Role of Tax Authorities..............................62

E. Taxpayer Privacy: The Case of Undocumented Taxpayers..................................64

CONCLUSION .......................................................................................................67 


\section{INTRODUCTION}

Blockchain technology was first outlined in the late 1980s by researchers who wanted to implement a system where a document's timestamps could not be tampered with. ${ }^{1}$ But it was not until almost two decades later that blockchain had its first real-world application with the launch of Bitcoin in $2009 .^{2}$ The Bitcoin protocol, or cryptocurrency more broadly, is built on blockchain, ${ }^{3}$ and blockchain is the original, underlying technology. ${ }^{4}$

Blockchain is a decentralized, immutable, peer-to-peer ledger. ${ }^{5}$ It is a newly emerged record keeping system, where digital information is recorded in each block of blockchain and managed by the group of users distributed in the network. It does not require a middleman to validate the information; instead, a consensus mechanism is used for each user distributed across the network to collectively validate the information. ${ }^{6}$ Such decentralization enables trust among the parties in the system and improves transparency, data immutability, security, and efficiency. With Bitcoin, the information recorded in the blockchain network consists of the transactions of Bitcoin. However, blockchain as a technology is capable of recording and managing any digital information and has applications beyond Bitcoin.

Recent applications of blockchain are elevating the technology above and beyond what cryptocurrencies are capable of. Blockchain is in the limelight when it comes to dealing with information and records in the digital era. Before blockchain, a centralized database management system was considered the solution for managing and exchanging information. Over time, it is becoming increasingly less safe to store everyone's information in a single central database because centralized databases are becoming targets for cyber-attacks and data breaches. Conversely, distributed ledger technology, or blockchain, is an alternative data management system with improved data integrity, immutability, and network resilience. In addition, it can protect the privacy of the users contributing data by paring it with critical security and cryptography. Because of these features, blockchain plays an

\footnotetext{
${ }^{1}$ Dylan Yaga et Al., BlockChain TeChnOlOGy OverviEW, NISTIR 8202, 2 (Oct. 2018), https://nvlpubs.nist.gov/nistpubs/ir/2018/NIST.IR.8202.pdf.

${ }^{2} \mathrm{Id}$. at 1 .

${ }^{3}$ In a research paper introducing the digital currency, Bitcoin's pseudonymous creator Satoshi Nakamoto referred to it as a new electronic cash system that's fully peer-to-peer, with no trusted third party. Satoshi Nakamoto, Bitcoin: A Peer-to-Peer Electronic Cash System 1, https://bitcoin.org/bitcoin.pdf.

${ }^{4}$ See infra Part I.C.

${ }^{5}$ YAGA ET AL., supra note 1 , at 1.

${ }^{6}$ Catherine Tucker \& Christian Catalini, What Blockchain Can't Do, HARV. Bus. REV. (June 28, 2018), https://hbr.org/2018/06/what-blockchain-cantdo?referral=03759\&cm_vc=rr_item_page.bottom.
} 
important role in helping institutions and governments around the world respond to the COVID-19 crisis, and blockchain technology is currently being integrated into healthcare systems and food supply chains. ${ }^{7}$ Because more data is being processed remotely and thus digitally, a data management system using blockchain is gaining more traction.

Despite blockchain's recent traction, Bitcoin and cryptocurrency more generally is often mistaken with blockchain. Even scholarly literature on blockchain conflates blockchain and cryptocurrency. ${ }^{8}$ Tax literature on blockchain is the same: mainly dealing with the nature of cryptocurrency for tax purposes and focusing on how users should comply with the tax system, while failing to actually discuss blockchain technology itself from a tax perspective. ${ }^{9}$ This historical confusion and the lack of a refined discussion on the broader concept of blockchain is understandable because cryptocurrency is the most famous and monetized product where individual taxpayers commonly face tax compliance issues.

Today though, the confusion and lack of refined discussion on blockchain is no longer justified given the new important role blockchain plays for managing and exchanging information in the "new normal." This Article goes beyond cryptocurrency to discuss how blockchain, or the distributed peer-to-peer ledger technology itself, can apply to the public sector. Specifically, this Article discusses how blockchain technology can be adopted by government actors in tax administration, including its limitations and what measures policymakers should consider in this process.

Blockchain is best suited for an area within the public sector that requires data redundancy, information transparency, data immutability, and a

\footnotetext{
${ }^{7}$ Nadia Hewett \& Rasmus Winther Mølbjerg, This Is How Blockchain Can Be Used In Supply Chains To Shape A Post-COVID-19 Economic Recovery, FORBES (June 19, 2020), https://www.forbes.com/sites/worldeconomicforum/2020/06/19/this-is-how-blockchaincan-be-used-in-supply-chains-to-shape-a-post-covid-19-economicrecovery/\#1a51e1f94c0e; Irving Wladawsky-Berger, Blockchain May Offer Solutions to Fighting Covid-19, WALL ST. J. (May 1, 2020), https://cointelegraph.com/news/howblockchain-technology-can-help-fighting-against-covid-19.

${ }^{8}$ See e.g., Carla L. Reyes, (Un)Corporate Crypto-Governance, 88 FORDHAM L. REV. 1875 (2020). But cf. Kevin Werbach, The Siren Song: Algorithmic Governance by Blockchain 2 n.5, in AFTER THE DigitAL TORNADO: NETWORKS, AlgORITHMS, HuMANITY (Kevin Werbach ed., 2020) (recognizing the difference of blockchain and cryptocurrencies and stating that the author uses "blockchain as a generic term for the collection of cryptocurrency, blockchain, and distributed ledger technologies.").

9 See e.g., Omri Marian, A Conceptual Framework for the Regulation of Cryptocurrencies, 82 U. CHI. L. REV. 53 (2015); Eric D. Chason, Cryptocurrency Hard Forks and Revenue Ruling 2019-24, 39 VA. TAX REV. 277 (2019); Abraham Sutherland, Cryptocurrency Economics and the Taxation of Block Rewards, 165 TAX NOTES 749 (2019).
} 
consensus mechanism. ${ }^{10}$ With these criteria, tax administration is a strong candidate to incorporate blockchain because it requires at least three of the four factors: data redundancy, information transparency, and data immutability. Certain areas of taxation also require the fourth factor, i.e., a consensus mechanism, because of the inherent lack of trust among the parties. In these areas, blockchain can be particularly helpful.

The first area where tax administration requires the first three factors is in payroll taxation. Tax administration is closely linked with collecting and managing tax information. A major goal of tax administration is to overcome the asymmetry of information between taxpayers and tax authorities. Tax information originates from various taxpayer activities, but it is not always readily available to the government who must acquire and process the information to enforce the tax system. Thus, people are required to share tax information with tax authorities via various routes. This information is sometimes self-reported by taxpayers, such as by filing tax and information returns, but often the information is reported by third parties including withholding agents or financial institutions, as is the case in the payroll tax. To overcome information asymmetry, the tax compliance system requires transparency and data immutability.

Tax information collected during tax compliance may also be shared with other tax authorities or institutions, and vice versa. In payroll taxation, the amount of wage is reported and shared with various government agencies and companies. ${ }^{11}$ The payroll system not only processes Social Security or Medicare taxes, but also withholds and pays federal, state, and local income taxes. Tax authorities, the Social Security Administration, and financial institutions collect the same information to process wage income amounts. Thus, the various systems impose significant burdens on the intermediaries (i.e., employers), and yet remain far from efficient because each government agency and institution holds their own register, in effect duplicating data already held by other institutions. Blockchain offers a better system addressing this inefficiency caused by data redundancy and offers transparency and data immutability.

The second area where tax administration requires all four factors is the exchange of tax information between multiple governments. The fourth factor of blockchain, a consensus mechanism, is a solution to the situation where parties in a peer-to-peer transaction do not fully trust each other, or where there is no central authority to validate transactions. This trust issue exists when a tax authority shares its information with other tax authorities.

\footnotetext{
${ }^{10}$ DEPARTMENT OF HOMELAND SECURITY (DHS), BlOCKCHAIN AND SUITABILITY FOR Government Applications, 2018 Public-Private Analytic Exchange Program 5 (2018).

${ }^{11}$ See infra Part II.C.1.
} 
If certain federal tax information is related to state and local taxation, then that information should be easily shared with state and local tax authorities, and vice versa. Currently, the federal government shares tax information with states and localities. While states should, and sometimes do, share with the federal government, they do so less robustly. The resulting information gaps are bad for tax administration. This is because the hierarchy between the federal government and state and local governments weakens when the two entities share information as peers. Blockchain enables a more robust exchange of information while respecting a more democratic relationship among the federal, state, and local governments. ${ }^{12}$

The same trust problem is even more conspicuous at the international level. ${ }^{13}$ If tax information is related to the tax jurisdictions of multiple countries, that information should be shared between the relevant countries. In the past decade, the need to fill cross-border information gaps have developed spurring the improvement of many information sharing systems, such as systems designed to facilitate the automatic exchange of information. However, none of these systems have succeeded in creating the real-time sharing of information, resulting in a significant time lag. Further, the systems remain too immature to fully monitor which information should be shared with what jurisdictions. The limitations of the systems is largely due to the lack of trust and a central authority in the global community, thereby making blockchain a compelling alternative.

Even before the rise of blockchain, tax administration has engaged in significant efforts to improve the system, propelled by a desire for greater efficiency, transparency, and better compliance to overcome the asymmetry of information. Tax authorities have attempted to collect and process information digitally, providing a more efficient environment for creating foolproof solutions and software. ${ }^{14}$ Taxpayers also expect that the process of taxpaying will become simpler, less costly, and less time-consuming and that their tax information will be properly used, stored securely, and protected from undue disclosure to unrelated parties or the public. The competing goals of tax administration, such as efficiency, transparency, simplicity, and taxpayer protection, generate constant tension and policy concerns. Are the competing goals of tax administration impossible to achieve collectively? Or is there an optimal solution available to balance the stated goals? While tax

\footnotetext{
${ }^{12}$ See infra Part I.C.3.

${ }^{13}$ See infra Part I.D.2.

${ }^{14}$ For example, Congress established the Electronic Tax Administration Advisory Committee through the IRS Restructuring and Reform Act of 1998 to meet the goal for electronic filing of tax and information returns. Electronic Tax Administration Advisory Committee (ETAAC), IRS, https://www.irs.gov/newsroom/electronic-tax-administrationadvisory-committee-etaac (last updated Jan. 11, 2021).
} 
administration has adopted various strategies to address these issues and questions, there is room for improvement.

Specifically, emerging technologies may solve this puzzle and contribute to the improvement of tax administration, considering that tax information is often collected and processed digitally these days. Blockchain is one of the most promising technologies to create a better system for managing digital tax information because of its ability to deliver reliable real-time information from many different layers to a large audience, as is the case with taxation. For this reason, this Article focuses on blockchain technology and explores the possibility of incorporating blockchain technology in tax administration.

This Article not only contributes to the scholarly analysis on the feasibility of incorporating blockchain in tax administration, but also offers a normative blueprint that policymakers and market players can refer to and, hopefully, readily adopt. Based on the author's extensive survey supported by the IRS Office of Chief Counsel and leading blockchain platforms, such as CoinBase and Ripple, this Article proposes a framework to help categorize areas of taxation in which blockchain would improve tax administration. ${ }^{15}$ The recommended areas of taxation are as follows: 1) reporting obligations of the same information to multiple tax authorities and agencies (e.g., payroll taxation, transfer pricing), 2) third-party reporting obligations (e.g., withholding tax), 3) transaction taxes (e.g., value added tax), and 4) information sharing (e.g., among federal, state, and local governments, and among multiple countries in international tax).

This Article suggests a private consortium blockchain, an evolution from the classic public blockchain, as the preferred structure for tax blockchain networks for the above areas. Parties in the blockchain consortium can trust each other without a third party because the data's immutability and decentralization ensures its integrity and network resilience, its confidentiality via encryption and access control, and its security. Moreover, a well-designed private consortium blockchain is effective in protecting taxpayer information from cyber-attacks and controlling who can access and share tax information. Thus, blockchain technology is capable of improving the existing tax administration's efficiency and transparency, while still maintaining taxpayer protection at the same time.

Applying blockchain to tax administration is not an impractical pipedream but can be adopted in the near future. Areas of the private sector that are closely related to tax administration, such as banking and financial services, have already adopted, or plan to adopt, blockchain technology. ${ }^{16}$ What is more, areas of the private sector that deal with information and record keeping, such as property and medical records, are also actively discussing

\footnotetext{
${ }^{15}$ See infra Part II.A.

${ }^{16}$ See infra Part I.C.1.
} 
incorporating blockchain technology. ${ }^{17}$ Since 2017, several projects sponsored by the federal government have begun analyzing the potential pros and cons of applying blockchain in the public sector. ${ }^{18}$ Notwithstanding these developments, there remains little study of applying blockchain in tax administration. This Article aims to fill this gap. This Article's analysis of blockchain designs and policy implications may also benefit broader audiences who are interested in diverse blockchain applications either in the private or public sector.

With that in mind, this Article provides normative considerations for policymakers deliberating blockchain initiatives in tax administration in several ways. First, the appropriate timeframe for blockchain implementation in tax administration depends on the timing of the widespread use of distributed ledger technology within many sectors of society. ${ }^{19}$ Despite some skepticism of blockchain technology being overhyped, an overwhelming majority of business executives expect that blockchain will eventually achieve mainstream adoption. ${ }^{20}$ So, it is wise to prepare for the next phase of blockchain development because the technology likely becomes readily available sooner rather than later.

Second, the areas of tax fit to incorporate blockchain are heavily intertwined with other sectors, such as financial institutions as well as other regulatory agencies and foreign governments. For streamlined performance, blockchain in tax administration should include interchangeable modules that connect with other sectors seamlessly. ${ }^{21}$ Standardization is also needed, but not at the price of harming innovation and competition by making the standards proprietary or less accessible.

Third, it is important to understand the limitations of blockchain for tax administration. Considering that the blockchain distributed ledger technology is the next phase of digital information management, the benefits of its application are limited to improving existing data management systems where information is already digitalized. It is uncertain how much the degree of the voluntary input of tax data by taxpayers at the intersection between offline and digital can be improved. For example, blockchain may not be effective in reducing the tax gap, much of which results from cash business, in the self-employment tax and the individual tax on business income. ${ }^{22}$

Finally, blockchain initiatives must be accompanied by additional

${ }^{17}$ See infra Part I.C.2.

18 Id.; Blockchain and Distributed Ledger Technology, OECD, http://www.oecd.org/daf/blockchain/.

${ }^{19}$ See infra Part III.A.

${ }^{20}$ Deloitte, DeloitTe Insights: Deloitte's 2020 Global BlockChain Survey 5 (2020) [hereinafter DELOITTE, SURVEY].

${ }^{21}$ See infra Part III.B.

${ }^{22}$ See infra Part III.C. 
legislation regulating the role of government and protecting taxpayers' rights and privacy. ${ }^{23}$ A properly designed blockchain has great potential to address the privacy concerns of taxpayers because it can systematically prevent the undue sharing of information, such as the sharing of undocumented taxpayers' information with other agencies or the cross-border sharing of information with hostile foreign countries. However, the proposed blockchain networks for tax administration are consortium networks, meaning that most individual taxpayers cannot participate in the network as a node. Only tax authorities, other agencies, certain withholding agents, and third-party reporters can participate in the network and serve as a node. This raises the concerns of who controls the information system and how to protect taxpayers' rights and privacy. One might assert that the government is a trustworthy administrator for a solution, but it might conflict with the nature of blockchain as a decentralized system. ${ }^{24}$ To truly be effective, blockchain must be accompanied by additional privacy legislation surrounding the control of tax information.

These policy implications may resonate with a broader audience beyond tax policymakers. Anyone who seeks a more efficient, transparent, and safer data management system can learn lessons from the blockchain applications explored in this Article as well as in the simulation of a tax blockchain system. The attempt to build a more democratic tax system among federal, state, and localities by adopting blockchain can inspire policymakers who struggle with federalism and state autonomy. Global leaders who have been hesitant to cooperate on many international administrative issues because of the lack of central authorities may welcome the idea that blockchain can offer a multilateral platform where information can be exchanged efficiently, and yet allow access to the information only to pre-selected parties, all executed automatically without the need of central administrator. To build upon this Article, scholars should explore other areas that can implement blockchain technology.

The remainder of this Article proceeds as follows. Part I overviews blockchain technology as a decentralized, immutable, peer-to-peer digital ledger. It introduces key features, important types of blockchain systems, and applications in the real world to shed light on the design of blockchain systems for tax administration. Part II evaluates the feasibility of incorporating blockchain in tax administration as well as provides policymakers with both criteria to consider in adopting blockchain and some recommended designs for blockchain networks. It also illustrates promising areas of taxation for blockchain initiatives, both in domestic and international

\footnotetext{
${ }^{23}$ See infra Parts III.D and E.

${ }^{24}$ This is so-called Vili's governance paradox of blockchain. See discussion infra Part III.D.
} 
tax. Part III offers normative considerations for policy makers deliberating blockchain initiatives for tax administration, such as the timeline, standardization, integration with other systems, limitations, and considerations for taxpayers' rights and privacy. The Article then concludes that the thoughtful application of blockchain would improve tax administration's efficiency and transparency while also still protecting taxpayers' information.

\section{UNPACKING BLOCKCHAIN}

For many, the term "blockchain" has become synonymous with Bitcoin, a cryptocurrency that has garnered significant public interest by challenging many of the norms generally associated with traditional currencies. ${ }^{25}$ While blockchain technology is utilized by Bitcoin, blockchain is a far broader technology than simply Bitcoin, or cryptocurrencies in general. Part I provides a primer on blockchain technology discussing the various types of blockchain systems, their applications in the private and public sectors, and how they are building blocks to expand blockchain's usage to tax administration.

\section{A. Blockchain Primer}

Although the media often highlights and publicizes stories on cryptocurrencies, what is far more significant is the technology behind cryptocurrencies, called blockchain. The best definition of blockchain is, "a peer-to-peer, distributed ledger that is cryptographically-secure, appendonly, immutable (extremely hard to change), and updateable only via consensus or agreement among peers (power of decentralization)." 26 This Subpart analyzes the definition and key features of blockchain technology.

\section{A Distributed, Immutable, Peer-to-Peer Ledger}

A blockchain is "a shared digital ledger of transactions between parties in a network, not controlled by a single central authority." 27 This shared digital

25 See e.g., Marco Iansiti \& Karim R. Lakhani, The Truth About Blockchain, HARV. BuS. REV. (Jan.-Feb. 2017), https://hbr.org/2017/01/the-truth-about-blockchain.

${ }^{26}$ IMRAN BASHIR, MASTERING BLOCKCHAin: Distributed LEDGER TECHNOLOGY, DECENTRALIZATION, AND SMART CONTRACTS EXPLAINED 16 (2nd ed. 2018).

27 Id. at 19; OECD, OECD BLOCKCHAIN PRIMER 4 (2019) [hereinafter OECD, BlocKCHAIN PRIMER]; HYPERLEDGER, AN INTRODUCTION TO HYPERLEDGER 4 (2018), https://www.hyperledger.org/wp-

content/uploads/2018/08/HL_Whitepaper_IntroductiontoHyperledger.pdf [hereinafter HYPERLEDGER, INTRODUCTION]. 
ledger is similar to a traditional record book or database. ${ }^{28}$ Like all ledgers, blockchain simply features a series of transactions or records. In the case of blockchain, these transactions take the form of individual "blocks," each of which is recorded on the distributed ledger. ${ }^{29}$ For example, if a person purchases a book from Amazon, information about the transaction, like the date, time, dollar amount of a purchase, and who is participating in the transaction is stored in the block.

Each independent block is incorporated into the chain by using a hashing system. ${ }^{30}$ In addition to the information about the transaction, each block also stores a unique code, called a "hash", that distinguishes one particular block from every other block. ${ }^{31}$ Each block is assigned its own unique hash, and it also contains the hash of the preceding block. ${ }^{32}$ When storing the digital information in the ledger, the block is added to the end of the blockchain in chronological order, as illustrated in Figure $1 .{ }^{33}$ Because each hash is mathematically connected to the data inputted, it would be impossible to change just one block without disrupting the mathematical formula. ${ }^{34}$

\section{Figure 1. Stylized Structure OF A BlockChaiN ${ }^{35}$}

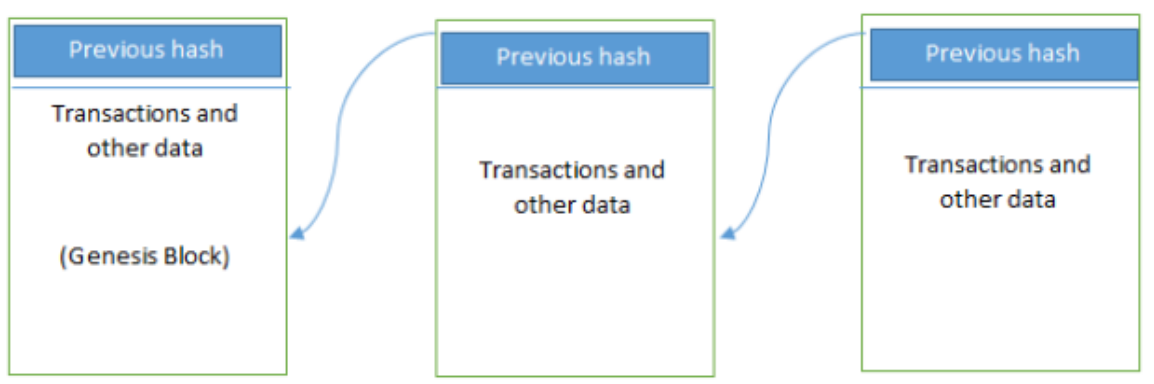

What makes blockchain unique from other ledgers in the current digital age is the fact that blockchain is "not controlled by a single central authority." 36 In traditional databases and information systems, data is stored

${ }^{28}$ OECD, BLOCKCHAIN PRIMER, supra note 27, at 4.

${ }^{29} \mathrm{Id}$.

${ }^{30} I d$.

${ }^{31} I d$. at 7 . Hashes are "a unique string of letters and numbers created from text using a mathematical formula." $I d$.

${ }^{32} I d$.

${ }^{33}$ BASHIR, supra note 26, at 17.

${ }^{34}$ OECD, BLOCKCHAIN PRIMER, supra note 27, at 7.

${ }^{35}$ BASHIR, supra note 26, at 20.

${ }^{36}$ OECD, BLOCKCHAIN PRIMER, supra note 27, at 4. 
on a centralized server that is owned and maintained by a central authority. ${ }^{37}$ Blockchain, on the other hand, adopts a distributed ledger system described in Figure 2. Rather than relying on a central authority to ensure the accuracy of the ledger, the blockchain relies on having identical copies of the ledger on the various user's computers that are geographically separated. These user's computers that contain a copy of the ledger are referred to as "nodes." 38 In Figure 3, each computer in the blockchain network is a node. Because there is an identical copy of the ledger stored on the various nodes, the accuracy is ensured by consensus protocol, (as discussed in more detail below). So, if the information on one computer were to be manipulated or changed, it would become apparent because all the other nodes sharing the blockchain would be inconsistent, and the nodes would reject that version of the ledger. ${ }^{39}$ This guarantees the immutability of the ledger. ${ }^{40}$

Figure 2. COMPARISON OF CENTRALIZED, DECENTRALIZED, AND DISTRIBUTED NETWORKS ${ }^{41}$
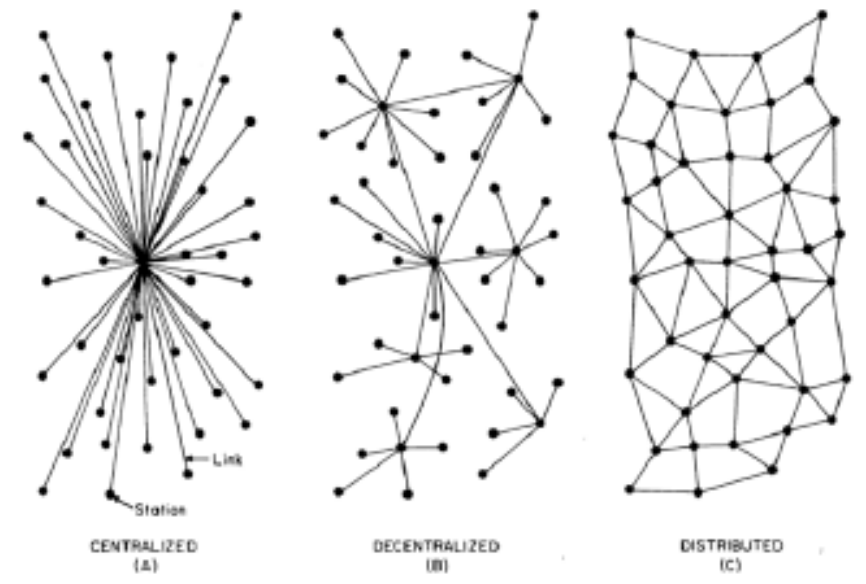

37 JAMIE BERRYHILL ET AL., BLOCKChaINS UnCHAINED: BLOCKCHAIN TECHNOLOGY AND ITS USE IN THE PUBLIC SECTOR 12, OECD (2018), available at https://www.oecdilibrary.org/governance/blockchains-unchained_3c32c429-en.

${ }^{38} I d$.

${ }^{39} I d$. at 7.

${ }^{40}$ BASHIR, supra note 26, at 24.

${ }^{41}$ PAUl BARAN, ON DisTRIBUTED COMMUNICATIONS: INTRODUCTION TO DisTRIBUTED Communications Networks, United States Air ForCe Project RAND 1-2 (1964); see also BERRYHILL ET AL., supra note 37, at 12. 
FiguRE 3. DistRIBUTED BLOCKCHAIN NETWORK

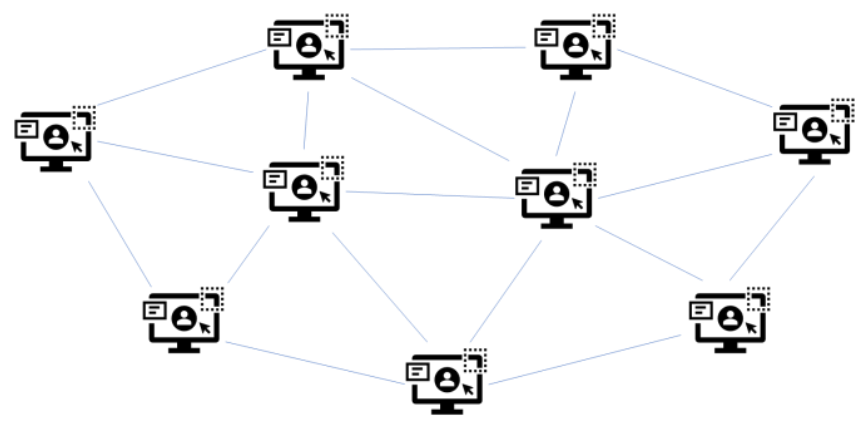

The process of how blockchain accumulates blocks is as follows. If one party requests a transaction, the requested transaction is funneled to a peerto-peer (or P2P) network (as illustrated in Figure 3) and broadcast to each individual computer (or node).$^{42}$ Individual nodes receive the request and validate the transaction using a consensus algorithm. Other public records of information, like stock exchanges, the DMV, or your local library, requires someone in charge to examine and validate the new data entries. Blockchain, however, does not require a trusted third party or intermediary that controls the ledger because a network of computers is in charge of that task. ${ }^{43}$ These networks often consist of thousands of computers spread across the globe. ${ }^{44}$ The network of computers in a blockchain system confirms the details of the information by a consensus mechanism. The approved transactions are represented as blocks and added to the blockchain ledger. Once the block is added to an existing chain, transactions are complete and permanent. Each computer in the blockchain network has its own copy of the blockchain. Although each copy of the blockchain is identical, spreading that information across a network of computers makes the information more difficult to manipulate. That is why blockchain is explained as a distributed, immutable, peer-to-peer ledger.

Consensus algorithms are the backbone mechanism that guarantees that information in the distributed ledger is always correct. ${ }^{45}$ Even if some of the nodes are likely to fail or to act dishonestly, a consensus system makes sure the information in the database is always correct by using pre-established

${ }^{42}$ Nakamoto, supra note 3 , at 3 .

${ }^{43}$ BASHIR, supra note 26, at 24.

${ }^{44}$ In the case of Bitcoin, this network consists of about 5 million computers or wallets across the globe. Garrick Hileman \& Michel Rauchs, Global CryptocurRency BENCHMARKING STUDY 8 (Cambridge Centre for Alternative Finance, 2017), https://www.jbs.cam.ac.uk/faculty-research/centres/alternative-finance/publications/globalcryptocurrency/\#.XxvQop5KiUk.

${ }^{45}$ BASHIR, supra note 26 , at 35 . 
rules based on the concept of Byzantine false tolerance. ${ }^{46}$ There are many different forms of consensus protocol in a blockchain system. ${ }^{47}$ For example, Bitcoin has adopted proof-of-work (POW) protocol, where the network nodes validate the information by competing among themselves to solve difficult math problems using their computer's processing power. ${ }^{48}$ If one node finds the correct answer and the majority of the nodes agree that such answer is correct, a consensus is achieved. For this work, the node receives rewards and rights to publish the new block associated with that work. ${ }^{49}$

The goal of the consensus protocol is not to make a perfect system; rather it aims to avoid the complete failure of the system. ${ }^{50}$ As a result, the system can continue operating even if some of the nodes fail or act maliciously. However, if the majority of the network decides to act maliciously, the system is susceptible to failures and attacks. This is the so-called the $51 \%$ attack on blockchain. ${ }^{51}$ For example, the POW and the proof-of-stake are susceptible to the $51 \%$ attack. ${ }^{52}$ In contrast, other types of consensus, such as proof of

\footnotetext{
46 The Byzantine fault tolerance deals with the Byzantine general's problem, which is a dilemma of how a group of Byzantine generals with each army situating in different locations may agree on a common decision as to either attack or retreat. (Putting this dilemma to the context of blockchain, each general represents a network node, and the needs need to reach consensus on adding a block to the chain.) The communication among the generals may be done through messages forwarded by a courier, but the message can get delayed, destroyed, or lost. In addition, some generals may act maliciously and send a fraudulent message. In order to prevent a total failure caused by this dilemma, the generals establish a rule ex ante, where i) each general has to decide as to attack or retreat, ii) after the decision is made, it cannot be changed, and iii) the action that the "majority" of the generals within such distributed network agree will be executed in a synchronized manner. Byzantine Fault Tolerance Explained, BINANCE ACADEMY (Dec. 20, 2020), https://academy.binance.com/blockchain/byzantine-fault-tolerance-explained.

${ }^{47}$ In addition to the POW, other types of consensus are called proof-of-stake, proof of burn, proof of activity, proof of capacity, proof of elapsed time, proof of authority, proof of importance, and Raft. BASHIR, supra note 26, at 37-39.

${ }^{48}$ Sutherland, supra note 9, at 754; Sarwar Sayeed \& Hector Marco-Gisbert, Assessing Blockchain Consensus and Security Mechanisms Against the 51\% Attack, 9 APPL. SCI. 6-7 (2019).

${ }^{49} I d$.

${ }^{50}$ BINANCE ACADEMY, supra note 46.

${ }^{51}$ See e.g., Sayeed \& Gisbert, supra note 48 (discussing broadly the danger of the majority of a system becoming susceptible to failure).

${ }^{52}$ For proof of stake (POS), users who want to participate in making and adding a block are required to lock a certain amount of its stake, such as coins, into the network. An algorithm chooses a node that will create, or forge in a technical terms, a block based on the user's stake; so the bigger the stake, the bigger the chances of a node to be selected as the next validator to forge the next block. If the network detects a fraudulent block, the forger node will lose its stake and right to participate as a forger in the future. However, POS is still vulnerable to the $51 \%$ attack. Sayeed \& Gisbert, supra note 48 , at 7-8.
} 
elapsed time ${ }^{53}$ and proof of activity, ${ }^{54}$ incorporate the feature of randomness in the protocol and reduce the chance of a $51 \%$ attack.

No matter what kind of consensus protocols they adopt, all blockchains rely on cryptography, which is a method of protecting information through the use of codes in hashing, so that it is difficult to decode by adversaries. ${ }^{55}$ Digital signatures and a certificate of authority protected by cryptography can provide basic identity protection in the blockchain network. ${ }^{56}$ There are also more advanced blockchain systems, such as private blockchain and permissioned blockchain discussed in Subpart B, which also offer confidentiality and privacy by combining both encryption and access control. ${ }^{57}$

\section{Blockchain's Key Features, Pros, and Cons}

Based on the overview above, key features of the blockchain system that distinguish it from existing ledger and database technology can be summarized as follows: blockchain systems are (1) distributed, (2) transparent enabling trust, and (3) immutable. Accordingly, it provides improved data security, network resilience, and efficiency.

Distributed: In a blockchain system, the database is maintained and held by all nodes distributed in the network, rather than being centrally located on a server or held by central authority. ${ }^{58}$ Any changes that are made to the ledger are agreed upon by all nodes in the network. ${ }^{59}$ Once the consensus is established, each node will update its own ledger. ${ }^{60}$ This distributed nature provides a level of reliability that a centralized and concentrated authority

${ }^{53}$ Proof of elapsed time, created by Intel for Hyperledger, is to decide the mining rights or the block winners based on a lottery system. Each participating node in the network is required to wait for a randomly chosen time period. The one which is assigned the shortest wait time wins the lottery and commits a new block to the blockchain. BASHIR, supra note 26 , at 38 .

${ }^{54}$ Proof of Activity (POA) tries to combine the best parts of POW and POS. The creation of block follows the POW mining process. Participating nodes must solve a mathematical problem with computing powers to create a new block. Then, the system is switched to POS, except that a group of validators are selected randomly. POA can prevent the chance of a $51 \%$ attach because it becomes impossible to predict who the validators would be in the future and the competition to hoard more coin among the participants prevents the computing power from being accumulated within a particular group of users. BASHIR, supra note 26, at 38.

\footnotetext{
${ }^{55} \mathrm{Id}$. at 61.

${ }^{56}$ BERRYHILL ET AL., supra note 37, at 47.

${ }^{57}$ HYPERLEDGER, INTRODUCTION, supra note 27, at 5.

${ }^{58}$ OECD, BLOCKCHAIN PRIMER, supra note 27, at 6.

${ }^{59} \mathrm{Id}$.

${ }^{60} \mathrm{Id}$.
} 
cannot.

Transparency and trust via consensus: Blockchain systems are engineered to enable direct, peer-to-peer transactions between parties who do not fully trust each other, or who do not trust any central authority to validate transactions or settle disputes. Even if the parties do not trust each other, they can trust the technology to record the information in a tamper-proof way. This makes the system transparent and, as a result, trust is established. Blockchain relies on a consensus mechanism to establish such trust. The consensus protocols formulate a set of rules that all nodes have agreed to follow and ensure that each node spread across the network adds the same new block. ${ }^{61}$ Because these protocols are implemented by code and can easily be tested by comparing an individual node's output against all the other nodes' in the system, it ensures trust between all users in the system.

Immutability: In a traditional database system, an authorized user can generally access, modify, and even permanently delete data stored on the database. On the other hand, data in blockchain is immutable. ${ }^{62}$ Once the data has been incorporated and recorded onto the blockchain, it is extremely difficult to go back and alter the contents of the block. ${ }^{63}$ Blockchain systems implement a hashing system that ties each block together in a series that cannot be disturbed without violating the remainder of the chain. ${ }^{64}$ For example, if a hacker were to attempt to edit your Amazon transaction, in order to change a single block (your block), the hacker would need to change each and every block after your block distributed across the entire blockchain. Recalculating all those hashes would take an enormous and improbable amount of computing power. Also, because the data contained on the blockchain is stored on thousands of independent nodes, changing any nodes will not affect the overall consensus. ${ }^{65}$

One of the most important advantages that blockchain systems provide over traditional databases and ledgers is reliability. Immutability and decentralization ensure data integrity and network resilience. ${ }^{66}$ Any attempt to alter the data on the blockchain creates discrepancy that other recordkeepers in the network immediately notice. The network then responds by shutting down the compromised node and removing it from the network. Thus, the accuracy of the data is guaranteed, and the distributed network eliminates the risk of a single-point attack, such as a DDoS attack. ${ }^{67}$ In

${ }^{61} I d$.

${ }^{62}$ BASHIR, supra note 26, at 24.

${ }^{63} \mathrm{Id}$.

${ }^{64}$ OECD, BLOCKCHAIN PRIMER, supra note 27, at 4.

${ }^{65} \mathrm{Id}$. at 6.

${ }^{66}$ BASHIR, supra note 26, at 25.

${ }^{67}$ Phillip Shaverdian, Start with Trust: Utilizing Blockchain to Resolve the Third-Party Data Breach Problem, 66 UCLA L. REV. 1242, 1278-79 (2019). 
addition, all transactions on a blockchain are cryptographically secured by the use of hashing, and thus, data security is provided. ${ }^{68}$ Furthermore, private blockchains offer both confidentiality and privacy by combining encryption and access control. ${ }^{69}$

By the same token, blockchain systems can be efficient. ${ }^{70}$ They can reduce cost due to the fact that blockchain does not require any central authority to maintain the system. In the past, every transaction or system that involved managing information required a middleman, like a bank, credit card company, or librarian, to confirm the identity of the relevant parties and validate the information. Requiring a middleman creates friction, delay, and expense. However, blockchain does not need a trusted third party or intermediary to validate transactions. Instead, a consensus mechanism is used to collectively validate transactions, enabling faster dealings, saving time, and reducing cost. ${ }^{71}$

Overall, the benefits of blockchain help create a better system for managing digital data. Parties in blockchain systems can trust each other without a third party. Blockchain's immutability and decentralization ensures data integrity and network resilience, confidentiality (encryption and access control), and data security.

Nonetheless, blockchain technology does offer some disadvantages that may diminish its value or applicability. First, blockchain systems, especially the type that uses the POW consensus protocol, consumes an enormous amount of energy. ${ }^{72}$ Because the system is utilizing the computing power of the thousands of nodes around the world, it ultimately ends up using more energy than a centrally located database. For example, the Bitcoin ledger used over 60 Tera Watthours in the past year. ${ }^{73}$ That is more energy than what is used in over 5 million American homes and is comparable to the energy consumption of entire countries, such as Kuwait. ${ }^{74}$

Second, for many of the same reasons, blockchain systems do not process transactions as quickly as is needed. This is the scalability problem. ${ }^{75}$ This limitation is evident in blockchain systems using the POW consensus protocol. ${ }^{76}$ It is not surprising given that a blockchain system is purposefully

${ }^{68}$ BASHIR, supra note 26, at 29.

${ }^{69}$ HYPERLEDGER, INTRODUCTION, supra note 27, at 5.

${ }^{70}$ BASHIR, supra note 26, at 25.

${ }^{71} \mathrm{Id}$. at 24-25.

72 BERRYHILL ET AL., supra note 37, at 32.

73 The most recent figures are available at Bitcoin Energy Consumption Index, DiGiCONOMIST (Aug. 2020), https://digiconomist.net/bitcoin-energy-consumption.

${ }^{74}$ BERRYHILL ET AL., supra note 37, at 32.

${ }^{75} \mathrm{Id}$. at 33 .

${ }^{76}$ For example, Bitcoin ledger can process only about seven transactions per second, 
redundant by carrying out identical computations on thousands of computers for the sake of reliability. Further amplifying the problem, the more a blockchain platform becomes popular and the more its users send information, the more it must be broadcasted throughout the entire growing blockchain network redundantly.

Third, in recent years, illegal activity has proven that people can also use blockchain's unique capabilities for harmful purposes. ${ }^{77}$ The FBI recently shutdown a blockchain system known as "silk road," an online darkweb marketplace, where criminals would take advantage of the confidentiality blockchain affords by making illegal purchases through the Bitcoin ledger. ${ }^{78}$ Although current U.S. regulation prevents users of online exchanges built on blockchain from full anonymity, ${ }^{79}$ this incident has alerted society of a downside that blockchain technology brings.

However, these challenges are mostly relevant to cryptocurrencies using POW consensus protocol, rather than the general blockchain technology behind cryptocurrencies. The challenges that are significant in some blockchain systems may not be significant in other systems with different designs. For example, the concerns about cryptocurrencies being used in illegal activities are not particularly relevant to a blockchain system that monitors food supply chains. The extreme energy consumption and scalability are a big problem for public blockchains using POW consensus protocol, but they would not be an issue for other blockchain platforms, especially private, permissioned ledgers using proof of authority consensus protocol. $^{80}$

Furthermore, the technical challenges of energy consumption and scalability are somewhat outdated because blockchain systems have evolved so quickly and significantly. Recent engineering and computer science literature in this topic has largely focused on how to overcome the challenges

whereas more traditional financial transaction system can process hundreds or thousands of transactions per second-e.g., Visa can process 1,667 transactions per second, and PayPal can 193 transactions per second. BERRYHILL ET AL., supra note 37, at 33.

77 Neel Mehta et Al., Blockchain Bubble or Revolution: The Present and FUTURE OF BLOCKCHAIN AND CRYPTOCURRENCIES 54-57 (2019).

${ }^{78} I d$. at 56. In the United States, online exchanges must obtain information about their customers when they open an account, verify the identity of each customer, and confirm that customers do not appear on any list of known or suspected terrorist organizations. AntiMoney Laundering (AML) Source Tool for Broker-Dealers, SECURITIES AND EXCHANGE COMMISSION (SEC), https://www.sec.gov/about/offices/ocie/amlsourcetool.htm (last visited Jan. 14, 2021).

${ }^{79}$ USA Patriot Act $§ 326(a)(2), 115$ Stat. 273, 317-18 (2001).

${ }^{80}$ For public sector applications of blockchain, permissioned blockchain systems with proof of authority consensus protocol is recommended. BERRYHILL ET AL., supra note 37, at 33. 
described. ${ }^{81}$ Thus, instead of weighing the pros and cons here, this Article will revisit the challenges and limitations of blockchain technology in Part III after exploring the application of blockchain in tax administration.

\section{B. Types of Blockchain System}

While all blockchain systems share the same core characteristics, not all blockchain systems are the same. Some of the most common variances that exist between systems are (1) public (or open) v. private (or closed) systems, depending on who can read and view the ledger, and (2) permissionless v. permissioned systems, depending on who can write and verify the ledger. ${ }^{82}$ After comparing these four types of blockchains, this Subpart introduces consortium blockchains, which is a noteworthy example of a private and permissioned blockchain system. Table 1 offers a summary of the types of blockchain systems.

\section{TABLE 1. TYPES OF BLOCKCHAIN SYSTEM}

81 How to overcome the technical challenges of blockchain, including energy consumption and scalability, often boils down to the question of how to improve the consensus protocol for various and evolving needs for blockchain. See e.g., Kyle Croman et al., On Scaling Decentralized Blockchains (A Position Paper) (2016), available at https://people.eecs.berkeley.edu/ dawnsong/papers/On\%20Scaling\%20Decentralized\%20 Blockchains_feb\%202016.pdf (diagnosing the scalability problem and proposing various solutions); BASHIR, supra note 26, at 561-81. Particularly for consortium blockchains that this Article recommends for tax administration infra Part II.B., many new consensus protocols have been developed. See e.g., Miguel Castro and Barbara Liskov, Practical Byzantine Fault Tolerance (1999), available at http://pmg.csail.mit.edu/papers/osdi99.pdf (introducing practical Byzantine Fault Tolerance (BFT) algorithm that work efficiently in asynchronous systems); Henrique Moniz, The Istanbul BFT Consensus Algorithm (2020), available at https://arxiv.org/pdf/2002.03613.pdf (presenting a Byzantine Fault Tolerant system to be used in the Quorum blockchain); Kejiao Li et al., Proof of Vote: A HighPerformance Consensus Protocol Based on Vote Mechanism \& Consortium Blockchain, 2017 IEEE 19TH INTERNATIONAL CONFERENCE ON HIGH PERFORMANCE COMPUTING AND COMMUNICATIONS (2017), available at 10.1109/HPCC-SmartCity-DSS.2017.61 (proposing a new consensus mechanism, called proof of vote, for consortium blockchains).

${ }^{82}$ Michele FincK, BlockChain Regulation AND GOVERnANCE IN EUROPE 14-16 (2019). 


\begin{tabular}{|c|c|c|c|c|c|}
\hline & & READ & WRITE & COMLIT & EXAMPLE \\
\hline \multirow[t]{2}{*}{ Public } & Permissionless & Anyone & Anyone & Anyone & $\begin{array}{l}\text { Cryptocurrency } \\
\text { e.g., Bitcoin, } \\
\text { Ethereum }\end{array}$ \\
\hline & Permissioned & Anyone & $\begin{array}{l}\text { Authorized } \\
\text { participants }\end{array}$ & $\begin{array}{l}\text { All or subset } \\
\text { of } \\
\text { authorized } \\
\text { participants }\end{array}$ & $\begin{array}{l}\text { Supply chain } \\
\text { ledger for retail } \\
\text { brand viewable } \\
\text { by public } \\
\text { e.g., Walmart } \\
\text { China }\end{array}$ \\
\hline \multirow[t]{2}{*}{ Private } & Consortium & $\begin{array}{l}\text { Authorized } \\
\text { participants }\end{array}$ & $\begin{array}{l}\text { Authorized } \\
\text { participants }\end{array}$ & $\begin{array}{l}\text { All or subset } \\
\text { of } \\
\text { authorized } \\
\text { participants }\end{array}$ & $\begin{array}{l}\text { Multiple banks } \\
\text { operating a } \\
\text { shared ledger } \\
\text { e.g., Quorum }\end{array}$ \\
\hline & $\begin{array}{l}\text { Private/ } \\
\text { Permissioned } \\
\text { "enterprise" }\end{array}$ & $\begin{array}{l}\text { Fully private } \\
\text { or restricted } \\
\text { to a limited } \\
\text { set of } \\
\text { authorized } \\
\text { nodes }\end{array}$ & $\begin{array}{l}\text { Network } \\
\text { operator } \\
\text { only }\end{array}$ & $\begin{array}{l}\text { Network } \\
\text { operator } \\
\text { only }\end{array}$ & $\begin{array}{l}\text { External bank } \\
\text { ledger shared } \\
\text { b/w parent co. } \\
\text { and subsidiaries } \\
\text { e.g., IBM } \\
\text { Blockchain } \\
\text { Platform }\end{array}$ \\
\hline
\end{tabular}

1. Public v. Private

Public (or open) blockchains are "open for anyone to read and view." 83 Under these systems, "anyone can download the entire ledger and view transaction data." 84 Conversely, private (or closed) blockchains can "only be viewed by a chosen group of people." 85 "These systems are not open for anyone to join and see." ${ }^{86}$ Instead, they require a gatekeeper to allow designated individuals to maintain a node on the network. ${ }^{87}$

Private blockchains are always permissioned allowing only a select group of users to write and verify the new block addition to the chain, and vice versa. Public blockchains are mostly permissionless blockchains, as observed in Bitcoin and other cryptocurrencies. However, public blockchains can be permissioned, in which case, anyone can read and view the ledger, but only authorized participants can write and verify the ledger. For example, a supply chain ledger of Walmart China may be viewed by the public, while only authorized suppliers may write and verify the ledger. ${ }^{88}$

${ }^{83}$ OECD, BLOCKCHAIN PRIMER, supra note 27, at 5.

${ }^{84}$ FINCK, Supra note 82, at 15.

85 OECD, BLOCKCHAIN PRIMER, supra note 27, at 5.

${ }^{86}$ FINCK, supra note 82 , at 15.

${ }^{87}$ Id.

${ }^{88}$ See infra Part I.C.1.d. 


\section{Permissionless v. Permissioned}

In addition to the distinction between public and private blockchains, blockchain systems also differ by being either permissionless or permissioned systems. Permissionless blockchains allow anyone to contribute by adding data to the ledger. ${ }^{89}$ In contrast, permissioned blockchains permit only a "select group of users to write (i.e. generate transactions for the ledger to record) and commit (i.e. verify new blocks for addition to the chain)." 90 Permissioned blockchains are often used by individual companies or groups of organizations, referred to as a consortium. $^{91}$

Permissionless blockchains are the traditional form of blockchain, open to anyone. Most cryptocurrencies, such as Bitcoin and Ethereum, adopt this form of blockchain. Because of the public nature of permissionless blockchains, anyone can read and write on the ledger. Hence, it may require significant cost to maintain the network. To prevent malicious interference, most permissionless blockchains use some form of a consensus system. ${ }^{92}$

On the other hand, for permissioned blockchains, users must be "authorized by some authority" to participate. ${ }^{93}$ The authority can be either an individual entity or a group of entities that verify admission based on an established set of rules. As a result, it is cheaper and more efficient to maintain the system, and such networks offer greater privacy among users. ${ }^{94}$

A unique feature of the permissioned blockchain is that it can restrict who can issue the transactions and who can access the ledger because only authorized users are participating in the network. ${ }^{95}$ This is not possible with permissionless blockchains. Furthermore, permissioned blockchains are flexible enough that a user can decide whether they want the public to see the content of the transactions or restrict it to authorized users only. ${ }^{96}$ Figure 4 illustrates the features of a permissioned, private blockchain, where only Parties A through $\mathrm{J}$ have permission to access. Each column represents

${ }^{89}$ FINCK, supra note 82, at 15; YAGA ET AL., supra note 1, at 5.

90 OECD, BLOCKCHAIN PRIMER, supra note 27 , at 4.

${ }^{91} I d$.

${ }^{92} I d$.

${ }^{93} I d$.

94 Jian Zhang, Deploying Blockchain Technology in the Supply Chain 4-5, INTECHOPEN (Jan. 17, 2019), https://www.intechopen.com/books/computer-security-threats/deployingblockchain-technology-in-the-supply-chain (discussing how permissioned blockchain allows for a "private blockchain" and often has "consensus protocols" that promote efficiency).

${ }^{95} \mathrm{Id}$.

${ }^{96} I d$. 
transacting parties and each row represents transactions. Note row 1, columns A and B, representing a transaction between A and B. Suppose C, D, and E are not relevant parties to this transaction, and the system wants to restrict their access to the information in block $[A \& B, 1]$. When block [A\&B, 1] is added, the transaction record is validated by and distributed to all parties, including $\mathrm{C}$ through $\mathrm{J}$. However, C, D, and $\mathrm{E}$ are restricted from accessing and viewing the record, while $\mathrm{F}$ through $\mathrm{J}$ may access and view the record.

Figure 4. Restricted ACCesS IN PERMisSIONED BLOCKCHAIN ${ }^{97}$

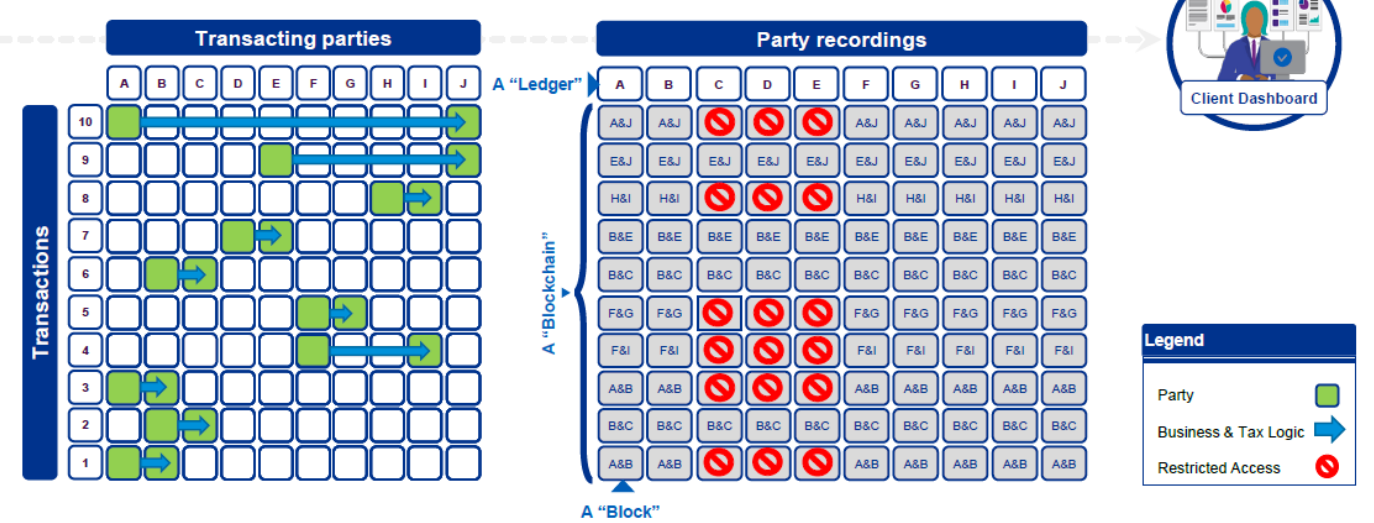

Both permissionless and permissioned blockchains have consensus models. However, the consensus process is much more efficient and costeffective in permissioned blockchains compared to permissionless blockchains because, in a permissioned blockchain, a certain level of trust already exists between the parties that are authorized to participate. ${ }^{98}$ Further, permissionless blockchains need some form of incentive to encourage participants to participate and ultimately validate the transactions, thereby requiring additional cost that is associated with compensating participants. ${ }^{99}$ Bitcoin is a good example. ${ }^{100}$ Conversely, permissioned blockchains are used by persons who share a common incentive for using and validating the blockchain. Hence, users in a permissioned blockchain do not expect or require any monetary form of compensation for their participation in the network.

${ }^{97}$ KPMG, BLOCKChain AND ARTIFICIAL INTELLIGENCE IN TAX 9 (Sep. 2019) (on file with author).

${ }^{98} I d$. at 5-6.

${ }^{99} I d$.

${ }^{100}$ To encourage persons to mine Bitcoin, the company is currently promising 12.5 Bitcoins per block that is processed. Clem Chambers, Cryptocurrency Mining Profits Are Way Down, Forbes (July 2, 2018), https://www.forbes.com/sites/investor/2018/07/02/cryptocurrency-mining-profits-are-waydown/\#1bdc50086c50. 
Because of this, a permissioned blockchain is a great option for organizations that want to work together, but do not fully trust each other. ${ }^{101}$ A permissioned blockchain is recommended for businesses enterprises and in other areas where a blockchain can be used to build up a trustworthy ledger or database in order to share information among relevant parties. Participants can benefit from the ability to "selectively reveal transaction information based on a blockchain network user[']s identity or credentials,"102 and to limit which of those users see the information in the transaction. For example, the IBM Blockchain Platform helps businesses to create their own private, permissioned blockchain. ${ }^{103}$

\section{Consortium Blockchain}

A noteworthy variation of the private blockchain system is a consortium blockchain. A consortium blockchain is a partially decentralized blockchain. Every node validates the list of transactions while only exposing the details of private transactions and contracts to relevant parties. The main difference between the plain-vanilla private blockchain and a consortium blockchain is who can write the transactions on the blockchain ledger. Both allows only authorized participants to read the ledger and view transaction data, because both are private blockchains. However, only the network operator or administrator can write and commit to the plain-vanilla private blockchain, whereas all (or at least a subset) of authorized participants may write and commit to the consortium blockchain.

Thus, a consortium blockchain enjoys the same benefits as a private blockchain by being functional, cost efficient, and private, without consolidating power in one user, thus offering the best of both worlds. As an example, JP Morgan has created a consortium blockchain called "Quorum" that aims to service the needs of a permissioned group of financial institutions. ${ }^{104}$ Because of the wide range of benefits and design options that are possible with consortium blockchains, this Article considers a consortium blockchain as one of the best options for tax administration as discussed further in Part II.B.

101 YAGA ET AL., supra note 1, at 6.

${ }^{102} I d$. (providing the example that a blockchain can show that a transaction between two parties occurred, but the content of the transaction is only visible to the involved parties).

103 See IBM Blockchain Platform, IBM, https://www.ibm.com/inen/blockchain/platform (last visited Jan. 14, 2021)

${ }^{104}$ See QUORUM, https://www.goquorum.com/ (last visited Jan. 14, 2021). 


\section{Applied Blockchains in the Private and Public Sectors}

Cryptocurrencies is the most famous application of blockchain technology. However, as previous Subparts explained, blockchain itself is a decentralized, immutable, peer-to-leer ledger technology with the benefits of transparency, immutability, and data security, which produces many applications across society. Recently, both the private ${ }^{105}$ and public sectors ${ }^{106}$ have begun to utilize blockchain technology as a data management system. This Subpart illustrates various applications of blockchain in both sectors, which provides insight for how blockchain design can benefit tax administration.

\section{Private Sector Applications}

The private sector has been some of the earliest adopters of blockchain technology, primarily in financial markets and services. Identifying these applications are helpful in determining how blockchain could benefit tax administration. They include cryptocurrency, banking and payment services, and general financial services.

\section{a. Cryptocurrency}

First, cryptocurrency. While blockchain has developed diverse applications in other areas, cryptocurrency is the earliest and the most wellknown application of blockchain technology. ${ }^{107}$ Since the launch of Bitcoin in January 2009, thousands of cryptocurrencies have emerged, including Ethereum, Tether, Bitcoin Cash, Libra, and Monero. ${ }^{108}$

The backbone of cryptocurrencies is to offer a digital currency that can operate without the need for central authority, and blockchain is their bedrock. ${ }^{109}$ Satoshi Nakamoto, who developed Bitcoin, explained that fiat currencies like the U.S. dollar are regulated and verified by a central

${ }^{105}$ E.g., financial services, supply chains, smart contracts, personal records.

${ }^{106}$ E.g., property records, voting, public health, defense, and compliance.

107 KEVIN WERBACH, THE BlOCKCHAIN AND NEW ARCHITECTURE OF TRUST 54 (2018).

108 Nathan Reiff, The 10 Most Important Cryptocurrencies Other Than Bitcoin, INVESTOPEDIA (Jan. 8, 2020), https://www.investopedia.com/tech/most-importantcryptocurrencies-other-than-bitcoin/. As of January 2021, there are over 4,000 cryptocurrencies tradable at CoinBase, one of the biggest digital exchange for cryptocurrency. See COINBASE, https://www.coinbase.com/price (last visited Jan. 14, 2021).

109 Satoshi Nakamoto, Bitcoin Open Source Implementation of P2P Currency, P2P FOUNDATION (Feb. 11, 2009), http://p2pfoundation.ning.com/forum/topics/bitcoin-opensource. 
authority, usually a bank or government. ${ }^{110}$ Under the central authority system, a user's data and currency are susceptible to the stability of their bank or government. If a user's bank collapses or they live in a country with an unstable government, the value of their currency is at risk. However, by spreading its operations across a network of computers, blockchain allows cryptocurrencies to operate without the need for such central authority. This not only reduces risk but also eliminates many of the processing and transaction fees. ${ }^{111}$ Cryptocurrencies also provide people during time of crisis or living in countries with unstable fiat currencies with a more stable currency that can apply to a wider network of individuals and institutions they can transact with, both domestically and internationally. ${ }^{112}$

Beyond cryptocurrency, blockchain technology is now being used across the financial industry, such as banking and post-trading processing, because blockchain can reduce costs and make transactions faster and more transparent. ${ }^{113}$

\section{b. Banking}

In banking, money transfer and payment services are actively considering blockchain. ${ }^{114}$ In traditional banking, depositing checks or sending money via wire transfers can take several business days. ${ }^{115}$ Once cleared, they can settle the amounts only during business days. The delay due to the mediation often exacerbates in cross-border payments. Conversely, blockchain never sleeps. By integrating blockchain, consumers can see their transactions processed in minutes, basically the time it takes to add a block to the blockchain, regardless of the time or day of the week. Beyond retail banking, banks also have the opportunity to exchange funds between institutions more quickly and securely. ${ }^{116}$ Because of the benefits of the technology, blockchain-based money transfer or payment services are being built upon either private or consortium blockchains in established companies ranging from JP Morgan to

\footnotetext{
${ }^{110} \mathrm{Id}$.

${ }^{111} \mathrm{Id}$.

${ }^{112}$ Marcia Narine Weldon \& Rachel Epstein, Beyond Bitcoin: Leveraging Blockchain to Benefit Business and Society, 20 TRANSACTIONS: TENN, J. BUS. L. 837, 845-46 (2019).

${ }^{113}$ Id. at 864; BASHIR, supra note 26, at 555.

114 See e.g., MATt HigGinson ET AL., BLOCKCHAIN AND RETAIL BANKING: MAKING THE CONNECTION, MCKINSEY \& COMPANY (June 7, 2019), https://www.mckinsey.com/industries/financial-services/our-insights/blockchain-and-retailbanking-making-the-connection\#.

115 HYPERLEDGER, INTRODUCTION, supra note 27, at 13 (discussing how bank regulations require verification of customers' identity for transactions).

116 Justin Pritchard, How Blockchain Is Changing Banking and Financial Services, THE BALANCE (Sep. 17, 2020), https://www.thebalance.com/how-blockchain-is-changingbanking-and-financial-services-4174354.
} 
the Ripple. ${ }^{117}$ Even central banks are implementing blockchain, ${ }^{118}$ with over 40 central banks implementing or researching blockchain technology. ${ }^{119}$ Some central banks have even implemented pilot programs based on Central Bank Digital Currency (CBDC), where "the central bank issues digital tokens on a distributed ledger that represent, and are redeemable for, central bank reserves in the domestic currency held in a separate account with the central bank." ${ }^{120}$ The CBDC program uses a private, permissioned blockchain network to limit participants, and access must be granted to participate and view transactions. ${ }^{121}$

Noting the growth of blockchain technology, there remains some hesitancy. PwC found that 57\% of those surveyed were unsure or unwilling to make use of this new technology. ${ }^{122}$ This hesitancy may be because of the newness of blockchain technology and the uncertainty that comes with change. ${ }^{123}$ However, Ripple argues that mindsets are changing and more are beginning to adopt blockchain technology. ${ }^{124} 59 \%$ of respondents in Ripple's report indicated that their organizations are in production or near production for payments-related use cases and 99\% indicate that their organization would consider using a digital asset as a means to instantly process cross-

${ }^{117}$ Santander with Ripple launched the first blockchain-based international money transfer service. One Pay FX: Blockchain for Streamlining International Transfers, SANTANDER (July 28, 2020), https://www.santander.com/en/stories/one-pay-fx-blockchainfor-streamlining-international-transfers. Similarly, Ripple offers RippleNet that connects hundreds of banks and payment providers to make payments with fiat currency or Ripple's own XRP cryptocurrency. RIPPLE, https://ripple.com/ripplenet (last visited Jan. 14, 2020); see also JP MORGAN, https://www.jpmorgan.com/onyx/liink.htm (last visited Jan. 14, 2021); Nathan DiCamillo, JPMorgan's Blockchain Lead Is Now in Charge of Ethereum-Based Interbank Information Network, CoINDESK (Oct. 8, 2020), https://www.coindesk.com/jpmorgans-blockchain-lead-is-now-in-charge-of-ethereumbased-interbank-information-network.

118 See BANK FOR INTERNATIONAL SETTLEMENTS (BIS), https://www.bis.org/about/index.htm?m=1\%7C1 (last visited Jan. 14, 2021).

${ }^{119}$ Christian Barontini \& Henry Holden, ProceEding With Caution - A Survey ON CENTRAL BANK Digital CURRENCY, BIS (Jan. 2019), https://www.bis.org/publ/bppdf/bispap101.pdf.

120 WORLD ECONOMIC FORUM, CENTRAL BANKS AND Distributed LEDGER TECHNOLOGY: HOW ARE CENTRAL BANKS EXPLORING BLOCKCHAIN TODAY? 5 (Mar. 2019), http://www3.weforum.org/docs/WEF_Central_Bank_Activity_in_Blockchain_DLT.pdf.

${ }^{121} \mathrm{Id}$.

122 PriceWaterhouseCooper, Financial Services Technology 2020 AND BEYOND: EMBRACING DISRUPTION (2020), https://www.pwc.com/gx/en/financialservices/assets/pdf/technology2020-and-beyond.pdf.

${ }^{123}$ William Girling, Blockchain: Optimizing Payments Or Disrupting Them?, FINTECH (Oct. 9, 2020), https://www.fintechmagazine.com/digital-payments/blockchain-optimisingpayments-or-disrupting-them.

124 RIPPle, RIPPLE'S BLOCKCHAIN IN PAYMENTS REPORT 2020 (2020), https://ripple.com/lp/blockchain-in-payments-report/. 
border payments. ${ }^{125}$ Blockchain is scalable in payment solutions, and as more organizations adopt a blockchain in payment systems, the integration will become easier. ${ }^{126}$ The COVID-19 pandemic helped get rid of some of the challenges in adopting blockchain because the modernization and streamlining of one's payment solution system became imperative when the entire world went remote. ${ }^{127}$

c. The Financial Industry

Other financial industries, especially capital markets dealing with debt and equity securities, have great hope for blockchain to restructure the clearing and settlement system. ${ }^{128}$

In capital markets, the post-trading process today suffers from significant bottleneck effect and duplication of effort, because every transaction must go through a fragmented workflow involving multiple parties in each step of process with different interfaces. To illustrate, if Parties A and B enter into a security transaction, such transaction must be validated, cleared, and settled. ${ }^{129}$ This process includes several steps that can involve a third partyusually a clearinghouse - to clear trades. Then the parties send separate settlement instructions to the settlement agent, and the settlement agent must collaborate to match the instructions. ${ }^{130}$ Then, custodians of the parties must adjust their position according to this transaction. On top of all this, there are reporting requirements to multiple regulatory and risk management entities, too. ${ }^{131}$ All these steps involving multiple parties with different interfaces require must then be reconciled "at the end of the business day." 132

On the other hand, a post-trade process with blockchain can be far more efficient. ${ }^{133}$ The blockchain protocol can verify the transaction between Parties A and B without a third party. Other relevant parties, including the regulatory agencies, also join the blockchain network and they receive the information on a need-to-know basis. All post-trade process is performed seamlessly without further duplicative reconciliation. The new system also

${ }^{125} \mathrm{Id}$. at 2.

${ }^{126} I d$. at 8 .

${ }^{127} I d$. at 20.

${ }^{128}$ Weldon \& Epstein, supra note 112, at 864-65.

${ }^{129}$ HYPERLEDGER, INTRODUCTION, supra note 27, at 13.

${ }^{130}$ HYPERLEDGER, INTRODUCTION, supra note 27, at 14.

${ }^{131}$ Clearing and Settlement in Major Market Countries, in Trading AROUnd tHE Clock: Global SECURities MARKeTS AND INFORMation TeChNOLOGY 81 (Princeton, 1990), https://www.princeton.edu/ ota/disk2/1990/9043/904310.PDF (describing how after clearance and settlement, "buy and sell data [is] reported by market participants.").

${ }^{132}$ HYPERLEDGER, INTRODUCTION, supra note 27, at 14.

${ }^{133} \mathrm{Id}$. 
makes the entire process in almost real time significantly reducing transaction time and cost. ${ }^{134}$

\section{Figure 5. COMPARISON OF Post-Trade Process With BlockCHAIN-BASED SYSTEM $^{135}$}
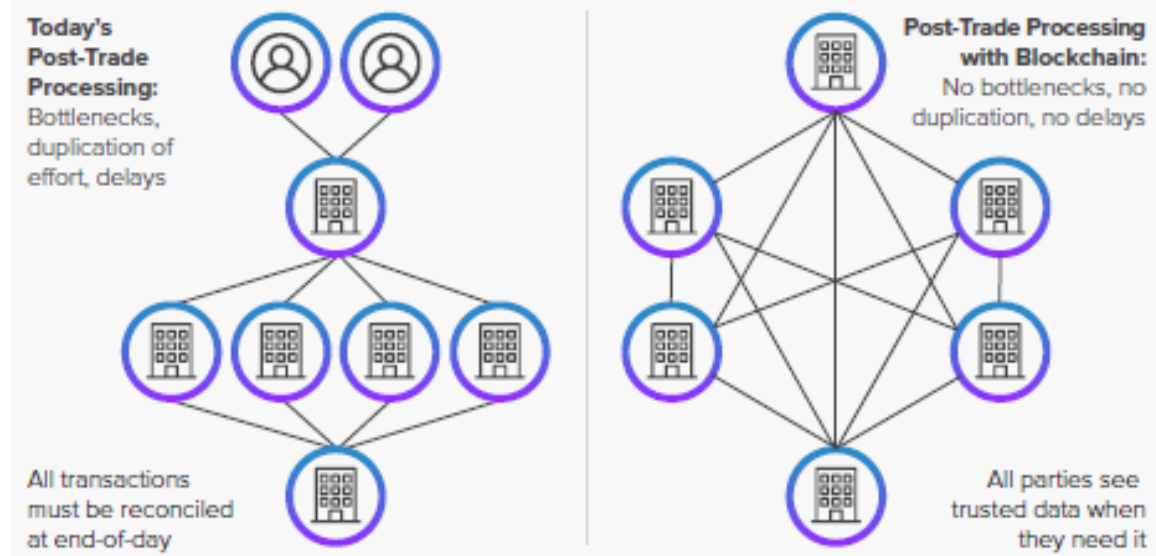

The Depository Trust \& Clearing Corporation (DTCC), the premier market infrastructure for the global financial services industry, aims to shift the post-trade clearing and settlement system to a consortium blockchain system with relevant parties as members. ${ }^{136}$ The DTCC thinks that blockchain is still immature to be fully incorporated into the post-trading process because of its problem with scalability and integration, among others. ${ }^{137}$ However, the DTCC continues to test the viability of blockchain by launching projects, such as a proof-of-concept blockchain to manage the netting process for repurchase agreement (repo) transactions and derivatives. $^{138}$

${ }^{134}$ Id.

135 Id. at 13.

${ }^{136}$ Press Release, Depository Trust and Clearing Corporation (DPCC), DPCC Unveils Proposals to Explore Digitalization in the Public \& Private Markets (May 18, 2020), available at https://www.dtcc.com/news/2020/may/18/dtcc-unveils-proposals-to-explorefurther-digitalization ("Project Ion explores whether the digitalization of assets and application of DLT can accelerate settlement and reduce cost and risk for the industry.").

${ }^{137}$ Joseph King, DTCC Sharpens Distributed Ledger Focus with White Paper, DTCC (Feb. 3, 2016), https://www.dtcc.com/news/2016/february/03/dtcc-sharpens-distributedledger-focus-with-white-paper.

${ }^{138}$ Press Release, DTCC \& Digital Asset Move to Next Phase After Successful Proofof-Concept for Repo Transactions Using Distributed Ledger Technology, DTCC (Feb. 27, 2017), available at https://www.dtcc.com/news/2017/february/27/dtcc-and-digital-assetmove-to-next-phase; Press Release, DTCC Selects IBM, Axoni and R3 to Develop DTCC's Distributed Ledger Solution for Derivatives Processing, DTCC (Jan. 9, 2017), available at 


\section{d. Supply Chains}

Non-financial firms can also apply blockchain to supply chains to improve productivity and efficiency. For example, Walmart has collaborated with IBM to introduce a blockchain system, called IBM Food Trust, to track the origin and travel of some of its perishable products. ${ }^{139}$ This effort is tied to an attempt to curb and quickly identify food-borne pathogens common to such products. Before the project, it could take days, if not weeks, to identify the source of food-borne illnesses. ${ }^{140}$ Because it is so hard to track the source of particular produce, governments commonly advise consumers to avoid products grown within a relatively large geographic area. As a result, millions of food items can be thrown out when an outbreak starts. ${ }^{141}$ But, if the source of a product can be effectively traced, companies will be able to react quickly and "only discard produce from the affected farms." 142

Walmart sees blockchain as a necessary tool in the supply chain of perishable foods because it provides traceability, immutability, and trustworthiness to the movement of the foods between differing parties. ${ }^{143}$ Each member of the perishable item's supply chain makes an entry on a blockchain ledger, "signing off when they receive it and then when they move it onto the next person in the chain." ${ }^{144}$ The initial pilot program showed great results, as Walmart was able to cut the time it took to track down the origin farm of mangoes "from 7 days to 2.2 seconds." 145 Walmart required all of its suppliers of fresh leafy greens to trace their products using the blockchain system by $2019,{ }^{146}$ and now it officially uses blockchain to "trace

https://www.dtcc.com/news/2017/january/09/dtcc-selects-ibm-axoni-and-r3-to-developdtccs-distributed-ledger-solution.

${ }^{139}$ HYPERLEDGER, HOW WALMART BROUGHT UNPRECEDENTED TRANSPARENCY TO THE FOOD SUPPLY CHAIN WITH HYPERLEDGER FABRIC (2019), https://www.hyperledger.org/wpcontent/uploads/2019/02/Hyperledger_CaseStudy_Walmart_Printable_V4.pdf [hereinafter, HYPERLEDGER, WALMART].

${ }^{140} \mathrm{Id}$. at 2.

${ }^{141} \mathrm{Id}$.

${ }^{142} I d$.

143 Id. at 3.

${ }^{144}$ Michael Corkery \& Nathaniel Popper, From Farmer to Blockchain: Walmart Tracks Its Lettuce, N.Y. TIMES (Sep. 24, 2018), https:/www.nytimes.com/2018/09/24/business/walmart-blockchainlettuce.html\#: :text=It\%20took\%20seven\%20days\%20for,for $\% 20$ food $\% 20$ safety $\% 20$ at $\% 2$ oWalmart.

${ }^{145}$ HYPERLEDGER, WALMART, supra note 139, at 5.

${ }^{146}$ Charles Redfield et al., Food Traceability Initiative Fresh Leafy Greens, WALMART NEWS RoOM, https:/corporate.walmart.com/media-library/document/blockchain-supplierletter-september-2018/_proxyDocument?id=00000166-088d-dc77-a7ff-4dff689f0001 (last visited Jan. 14, 2021). 
the origin of over 25 products from 5 different suppliers." 147 Walmart's technical partner, IBM, has been offering the IBM Food Trust to a broader circle of food industry, including more than 80 members and tracking over 1,300 products. ${ }^{148}$

The Walmart-IBM system is a private permissioned blockchain, where a member company can view products' history, location, certifications, tests, and temperature data only if such firm is permissioned to access the data. ${ }^{149}$ However, customers cannot access the detailed information of the food safety and quality management. Given that one of the strong motivations to adopt blockchain in food supply chain is to improve transparency of the data, keeping the public from the data does not fulfill the goal of the project.

Walmart China made an improvement on this point. Its new blockchain platform introduced in 2019 is using a hybrid blockchain-a public and permissioned blockchain. ${ }^{150}$ Thus, Walmart China can transact with suppliers on a permissioned blockchain ledger, while sharing information about products with consumers is also available thanks to its feature as a public ledger. Customers can scan QR codes with dozens of products to discover their origin and authenticity. ${ }^{151}$ Amid the COVID-19 pandemic, the supply chains in the food industry have required traceability and transparency even more not only for the industry but also the customers, such as restaurants and general public. Thus, the food industry may need to embrace public and permissioned blockchains more actively than now.

\section{e. Smart Contracts}

A smart contract is a computer code that can be built into the blockchain to facilitate, verify, or execute a contract automatically without human intervention. ${ }^{152}$ Smart contracts are computer programmed rules stating "ifthen" logic. They do not necessarily need a blockchain to run. However, due to the benefits that blockchain can offer, smart contracts are mostly executed

\footnotetext{
${ }^{147}$ HYPERLEDGER, WALMART, supra note 139 , at 1.

148 Biser Dimitrov, How Walmart And Others Are Riding A Blockchain Wave to Supply Chain Paradise, FORBES (Dec. 2019), https://www.forbes.com/sites/biserdimitrov/2019/12/05/how-walmart-and-others-areriding-a-blockchain-wave-to-supply-chain-paradise/?sh=732c97917791. Other notable members are Carrefour, Driscoll's, McLane, Kroger and Tyson.

${ }^{149}$ IBM, ABOUT IBM FOOD TRUST 3-5 (2020).

${ }^{150}$ Daniel Palmer, Walmart China Teams with VeChain, PwC on Blockchain Food Safety Platform, COINDESK (Jun. 25, 2019), https://www.coindesk.com/walmart-chinateams-with-vechain-on-blockchain-food-safety-platform. Walmart China is teamed up with VeChain, PwC and others, and not with IBM.

${ }^{151} I d$.

152 David J. Shakow, The Tao of The DAO: Taxing an Entity That Lives on a Blockchain,
} INST. L. ECON. 4 (2018). 
on blockchain platforms. ${ }^{153}$ If a set of conditions in smart contracts that the users agreed to are met, the terms of the agreement are automatically executed. $^{154}$

For example, Amy is renting Ben her apartment using a smart contract. ${ }^{155}$ Amy agrees to give Ben the door code to the apartment as soon as Ben pays Amy his security deposit. Both parties separately would send their portion of the deal to the smart contract, which would hold onto and automatically exchange Amy's door code for Ben's security deposit on the date of the rental. Both Ben and Amy can know when the commitments are fulfilled transparently without delay and there is no need to confirm the receipt of payment or send the door code separately. If Amy does not supply the door code by the rental date, the smart contract refunds Ben's security deposit. This eliminates the risk of delays and reliance on middlemen to follow through on their commitments. Also, the information sharing between parties is transparent, time-stamped, and irreversible.

Because of these unique features, smart contracts have benefits that especially are significant to the financial industry. ${ }^{156}$ In addition, blockchain's smart contract feature may further develop or enable decentralized autonomous organizations (DAOs), where corporate governance and operations can be executed by computer codes automatically. ${ }^{157}$

\section{f. IDs and Personal Data Management}

Blockchain is essentially a data management system. One area that it contributes to is managing personal information and records securely. ${ }^{158}$ Existing data management systems might protect personal data with encryption and security protocol, but they remain susceptible to cyberattacks. Blockchain offers enhanced security because of its distributed and immutable nature, and at the same time an individual owner of the data can control who has access to the data. ${ }^{159}$

The blockchain industry has developed portable digital identities for this purpose. ${ }^{160}$ Such digital identities are protected by cryptography in

${ }^{153}$ BASHIR, supra note 26 , at 53-54.

${ }^{154} I d$. at 54 .

155 This example is based on the explanation in Luke Conway, Blockchain Explained, INVESTOPEDIA (last updated Nov. 17, 2020), https://www.investopedia.com/terms/b/blockchain.asp.

156 BASHIR, supra note 26, at 261.

157 WERBACH, supra note 107, at 68.

158 See e.g., HYPERLEDGER, INTRODUCTION, supra note 27, at 17.

${ }^{159} \mathrm{Id}$.

160 See e.g., Id. 
blockchain protocol and can offer rich metadata of an individual's identity and sophisticated access control. ${ }^{161}$ Those digital identities are shared via the blockchain so that individuals may use their virtual identity wherever the blockchain is accepted. This feature benefits financial data management as well. Financial institutions are required to manage customer data while also having to abide by the laws and regulations that prevent money laundering and terrorist support. ${ }^{162}$ Such data management is expensive and even more challenging if financial institutions must comply with different international regulations for cross-border transactions. ${ }^{163}$ However, blockchain is borderless. The digital identities and financial records managed via blockchain can reinvent the financial record management system.

Additionally, even healthcare patients can leverage blockchain to securely store their medical records and regulate access, thereby ensuring privacy. ${ }^{164}$ When a medical record is generated and signed, it can be written into the blockchain with a private key, which provides patients with confidence that the record cannot be changed and is only accessible by certain individuals or organizations, such as their medical providers. ${ }^{165}$ Furthermore, blockchain could reinvent the way a patient's electronic health records are shared among medical providers. ${ }^{166}$ In traditional health record management systems, each institution silos their patients' data, resulting in fragmentation and an inefficient data sharing mechanism. This results in inefficient care coordination during medical emergencies due to the lack of critical medical information. ${ }^{167}$ However, as pointed out above, blockchain can offer safer mechanisms for the health industry's exchange of medical data.

\section{Public Sector Applications}

The primary benefits that Blockchain can offer - that is, promoting trust and greater transparency about data management-holds promise to benefit the public sector as well. Many countries are considering using blockchain in government settings in various ways, and the number of projects is growing. There were only 117 initiatives in 26 countries for using blockchain in the

${ }^{161} I d$.

162 Colleen Baker \& Kevin Werbach, Blockchain in Financial Services, in FINTECH: LAW AND REGULATION X.24-25 (2019).

163 Id. at X.26.

${ }^{164}$ Ray Hales Hylock \& Xiaoming Zeng, A Blockchain Frameworkfor Patient-Centered Health Records and Exchange (HealthChain): Evaluation and Proof-of-Concept Study, 21 J. MED. INTERNET RES. 1 (2019), available at https://www.jmir.org/2019/8/e13592/pdf.

${ }^{165} \mathrm{Id}$.

166 Id. at 2 .

${ }^{167} \mathrm{Id}$. 
public sector in 2017; in 2018, there were 202 initiatives in 45 countries. ${ }^{168}$

In the United States, the General Service Administration (GSA) launched the Emerging Tech Atlas program in 2017 to study the benefits that emerging technologies, including artificial intelligence and blockchain, may bring to public services. ${ }^{169}$ Since then, many federal, state, and local working groups have explored the potential applications of blockchain in the public sector to promote trust and integrity in government. ${ }^{170}$ Below are some of the notable initiatives.

\section{a. Property Records}

Today, the process of recording property rights in a local recording office is both burdensome and inefficient. A physical deed must be delivered to a government employee at the local recording office, where it is manually entered into the county's central database and public index. ${ }^{171}$ In the case of a property dispute, claims to the property must be reconciled with the public index. ${ }^{172}$

This process is not just costly and time-consuming. It is also riddled with human error, where each inaccuracy makes tracking property ownership less efficient. Blockchain has the potential to eliminate the need for scanning documents and tracking down physical files in a local recording office and transform the process of recording property rights. ${ }^{173}$ If property ownership is stored and verified on the blockchain, owners will be able to trust that their deed is accurate and permanent without having to deal with the burdensome current process of authenticating deeds. ${ }^{174}$

Noting the potential benefits, Cook County, Illinois, piloted a program in

\footnotetext{
168 Observatory of Public Sector Innovation, Blockchain and its Use in the Public Sector 17-18 (June 20, 2018), https://oecd-opsi.org/wp-content/uploads/2018/06/BlockchainsUnchained-Slides.pdf.

${ }^{169}$ GSA Blog Team, GSA Launches Emerging Tech Atlas to Encourage Collaboration, U.S. GENERAL SERVICES AdMINISTRATION (Oct. 25, 2017), https://www.gsa.gov/blog/2017/10/25/GSA-Launches-Emerging-Tech-Atlas-to-EncourageCollaboration.

${ }^{170}$ See, e.g., United STATES AGENCY for InTERnational DeVelopment, USAID PRIMER ON BLOCKCHAIN 5 (Apr. 27, 2018), available at https://www.usaid.gov/digitaldevelopment/digital-finance/blockchain-primer (discussing the ability of blockchain technology to be more "relevant" in "environments of incomplete trust" and how a "lack of trust, whether in people or in systems people rely on, will always be an underlying driver.").

${ }^{171}$ Weldon \& Epstein, supra note 112, at 869.

${ }^{172} \mathrm{Id}$.

${ }^{173} I d$.

${ }^{174} I d$.
} 
2017 to record the county land registry on a blockchain. ${ }^{175}$ The program identified the potential application of the various features of blockchain technology, such as the decentralized ledger, to improve access to house titles and other verifiable property data. The pilot program concluded in 2017 with meaningful lessons in the final report, stating that some aspects of blockchain worked for the property recording system while some did not, and that the government may implement certain aspects of blockchain individually or selectively. ${ }^{176}$ Furthermore, the report suggests that "if the use of blockchain were to be extended to the maintenance of a records system, it would be most optimal if the record-keeping ledger were to be distributed across all land records offices in Illinois, allowing economies of scale and the ability to create true distributed consensus." 177

\section{b. Voting}

Voting is still executed in a low-tech method despite this digital age, because security is far more important to the public and federal, state, and local legislative branches than efficiency is. ${ }^{178}$ Consequently, our low-tech voting process is susceptible to many errors, such as hanging chads and miscounts. ${ }^{179}$ Voting with blockchain carries the potential to eliminate election fraud and boost voter turnout, as was tested in the 2018 midterm elections in West Virginia as an alternative to mailed absentee ballots. ${ }^{180}$ Each vote would be stored as a block on the blockchain, making the cast votes nearly impossible to tamper with. A voting blockchain would create an atmosphere of transparency and trust in the electoral process, reducing the personnel needed to conduct an election and provide officials with instant results.

Encouraged by West Virginia's experience, the City of Denver and Utah County also planned to implement a pilot program to offer blockchain-based

\footnotetext{
175 John Mirkovic, Blockchain Pilot Program Final Report, COOK COUNTY RECORDER OF DEEDS 7 (2017), http://cookrecorder.com/wp-content/uploads/2016/11/Final-ReportCCRD-Blockchain-Pilot-Program-for-web.pdf.

${ }^{176} I d$. at 4.

177 Id.

178 Sarah Holder, Is This Experiment in Digital Democracy Too Crazy to Work, BlOOMBERG CiTYLAB (Sep. 11, 2018), https://www.bloomberg.com/news/articles/2018-0911/blockchain-based-voting-is-coming-to-west-virginia.

${ }^{179}$ See e.g., Samantha Levine, Hanging Chads: As the Florida Recount Implodes, the Supreme Court Decides Bush v. Gore, U.S. News (Jan. 17, 2008), https://www.usnews.com/news/articles/2008/01/17/the-legacy-of-hanging-chads.

180 Aaron Mak, West Virginia Introduces Blockchain Voting App for Midterm Election, SLATE (Sep. 25, 2018), https://slate.com/technology/2018/09/west-virginia-blockchainvoting-app-midterm-elections.html.
} 
voting service to active duty military in the coming elections. ${ }^{181}$ West Virginia passed a law requiring an electronic voting option for counties across the state. ${ }^{182}$ However, it later decided in February of 2020 not to use the blockchain-based voting option after the audits by MIT and the Department of Homeland Security (DHS) revealed security concerns regarding the particular mobile app used by West Virginia. ${ }^{183}$

Despite the controversy over the current flaws in blockchain voting systems, the need for secure remote voting increases in the wake of COVID19. The U.S. Postal Service (USPS) filed a new patent in 2020 to use blockchain to make mail-in voting as a safe alternative to traditional physical voting. ${ }^{184}$

\section{c. Public Health}

Important developments of blockchain applications occurred in connection with the public health crisis. Since 2017, the Centers for Disease Control (CDC) launched several projects to establish better public health surveillance. ${ }^{185}$ Those projects aim to improve continuous and systemic collection, analysis, and interpretation of health-related data to respond to public health crises. ${ }^{186}$ Tracking major public health developments requires to collect and process tremendous amount of data and address the privacy and security concerns at the same time. The CDC projects would allow the agency

${ }^{181}$ Nikhilesh De, City of Denver to Pilot Blockchain Voting App in Coming Elections, COINDESK (Mar. 7, 2019), https://www.coindesk.com/city-of-denver-to-pilot-blockchainvoting-app-in-coming-elections; Daniel Palmer, Utah County to Offer Blockchain Voting App in Municipal Elections, COINDESK (July 23, 2019), https://www.coindesk.com/utahcounty-to-offer-blockchain-voting-app-in-municipal-elections

${ }^{182}$ Kevin Collier, West Virginia Plans to Make Smartphone Voting Available to Disabled People for 2020 Election, NBC NEWS (Jan. 31, 2020), https://www.nbcnews.com/tech/security/west-virginia-plans-make-smartphone-votingavailable-disabled-people-2020-n1127931.

${ }^{183}$ Samuel Haig, West Virginia Abandons Blockchain Voting in Favor of Paper, COIN TELEGRAPH (Mar. 2, 2020), https://cointelegraph.com/news/west-virginia-abandonsblockchain-voting-in-favor-of-paper; Danny Nelson, MIT Wasn't Only One Auditing Voatz - Homeland Security Did Too, With Few Concerns, CoINDESK (Feb. 13, 2020), https://www.coindesk.com/mit-wasnt-only-one-auditing-voatz-homeland-security-did-toowith-fewer-concerns. West Virginia used the voting app developed by Voatz. MIT researchers found that hackers could alter, stop or expose how an individual user has voted, and that Voatz's use of a third-party vendor for voter identification and verification raises privacy concerns.

184 Benjamin Pirus, US Postal Service Files Blockchain Voting Patent Following Trump Cuts, CoIN TELEGRAPH (Aug. 14, 2020), https://cointelegraph.com/news/us-postal-servicefiles-blockchain-voting-patent-following-trump-cuts.

${ }^{185}$ DHS, supra note 10, at 29.

${ }^{186} I d$. 
to quickly respond to health crises as they unfold.

The CDC projects also explore the application of blockchain in many levels, such as within CDC, between CDC and partners in the private sector, such as health providers, financial institutions, and food suppliers, and a consortium blockchain among the entire public health community, including federal, state, and local governments. ${ }^{187}$ The approach to create a consortium blockchain resonates with the tax administration considering blockchain initiatives, which will be further discussed in Part II.B.

In the wake of COVID-19, the first real world application in the public sector emerged to respond to the public health crisis. In July 2020, the U.S. Department of Health and Human Services (HHS) took control over COVID19 data reporting from the CDC and launched a COVID-19 patient data tracking system, called the HHS Protect. ${ }^{188}$ The HHS Protect uses blockchain technology to ensure that the data for COVID-19 hospitalizations is accurate, transparent, and more easily traceable. ${ }^{189}$ It is interesting to see that the HHS Chief Information Officer Jose Arrieta had to explain to the public and media that the blockchain the HHS uses is not like Bitcoin or Ethereum or that of "anarchists and disruptors," and that the true nature of blockchain is ensuring data immutable and sharing and tracing such data. ${ }^{190}$

Blockchain is also being discussed as a possible solution for transparent and safe distribution of COVID-19 vaccines. ${ }^{191}$ Before the COVID-19 pandemic, the Food and Drug Administrations (FDA) has launched a blockchain project, called Real-Time Application for Portable Interactive Devices (RAPID) as a tool to manage data for therapeutic drug interventions employed during public health crisis. ${ }^{192}$ This project can facilitate the real-

\footnotetext{
${ }^{187}$ Brian Lee, Director of the Informatics Innovation Unit in the Division of Public Health Information Dissemination, February Presenter at the CDC Health Information Innovation Consortium, at 33 (Feb. 20, 2018), available at https://www.cdc.gov/ddphss/chiic/forums/CDC-Blockchain-Overview_-v16_tgs_2_2018508.pdf.

188 Jason Brett, U.S. Health Department Chief Discloses Functioning Blockchain Project To Track Covid-19, FORBES (July 24, 2020), https://www.forbes.com/sites/jasonbrett/2020/07/24/us-health-department-chief-disclosesfunctioning-blockchain-project-to-track-covid-19/?sh=7e4bf7991949.

${ }^{189} I d$.

${ }^{190}$ Brett, supra note 188. Arrieta is a blockchain expert. While at GSA, Arrieta was one of the core members of a blockchain working group to study potential blockchain applications in various agencies in the U.S. government.

${ }^{191}$ Peter Fretty, FDA Approval Is Not The Only Vaccine Challenge, INDUSTRYWEeK (Dec. 9, 2020), https://www.industryweek.com/covid19/article/21149716/fda-approval-isnot-the-only-vaccine-challenge.

192 DATA FoundATION, BRINGING BlOCKCHAIN INTO GOVERNMENT: A PATH FORWARD FOR CREATING EFFECTIVE FEDERAL BlockChain INITIATIVES 11 (2019).
} 
time exchange of data on vaccine distribution and possible harmful effects among agencies and medical providers without requiring the data to go through the central database that often results in a bottleneck effect. ${ }^{193}$ Inspired by the existing efforts, the medical industry expects that blockchain could help resolve the concerns relating to the distribution and management of the COVID-19 vaccines. ${ }^{194}$ With the use of blockchain, pharmaceutical companies can show step-by-step details of the manufacturing, distribution, and transportation of the vaccines, such as transportation temperature and vaccine life cycle. ${ }^{195}$ Furthermore, blockchain can benefit vaccine suppliers and distributers as well as regulators by offering a tool to monitor fraudulent products and any harmful effects associated with treatment. ${ }^{196}$

d. More Examples

In addition to the areas explored above, several working groups and pilot projects are discussing possible blockchain applications in the public sector.

A project launched by the U.S Citizenship \& Immigration Services (USCIS) and the Department of Labor (DOL) shows a potential to use blockchain among multiple government agencies. ${ }^{197}$ The Temporary Work Visa Program requires both the DOL and USCIS to gather and verify information, respectively, which requires significant and redundant paperwork for workers and employers. The two agencies launched a blockchain project to streamline the Temporary Work Visa process between them, hoping to "increase interoperability between agencies, allowing separate agencies (e.g., USCIS and DOL) to communicate more transparently, while permitting granular control of the permissions on shared information by making certain fields visible to some users and restricting access to others."198

Multiple projects are hoping to use blockchain as a secure supply chain program in the government sector. For example, the Department of Treasury launched a pilot program using blockchain to track and manage governmentowned inventory, such as computers and cell phones. ${ }^{199}$ A more interesting project is considered by the Department of Defense, combining blockchain and 3-D printing technology to produce on-demand fabrication of military equipment at military facility located worldwide. ${ }^{200}$ Suppose that an offshore

\footnotetext{
${ }^{193} I d$.

${ }^{194}$ Fretty, supra note 191.

${ }^{195} I d$.

${ }^{196} \mathrm{Id}$

${ }^{197}$ DHS, supra note 10, at 29.

${ }^{198}$ Id.; DATA FOUNDATION, supra note 192, at 9-10.

${ }^{199}$ Id. at 14; DHS, supra note 10, at 29.

${ }^{200}$ Id. at $32-33$.
} 
military maintenance facility needs aircraft components but faces logistical challenges to timely receive the replacement parts. ${ }^{201}$ With $3-\mathrm{D}$ printing technology, the logistical challenges can be resolved if the manufacturer offers design information and files so that the maintenance facility can print those parts on-site. However, the interests of the military consumers and that of the producers are not aligned. The military consumers wish to have confidence that the printed parts are a true representation of the original specification and have not been tampered with by adversaries, whereas the commercial manufacturers are more concerned about appropriate compensation on each printing. In this situation where immutability of information is critical and parties who do not fully trust each other, the employment of blockchain can lead to a good solution to guarantee the immutability, transparency, and security of the transaction.

Several projects take advantage of the blockchain networks in the crossborder context. The U.S. State Department is exploring projects to use blockchain to fight forced labor, child labor, human trafficking, third-country workers and shipping fraud, and other illegal practices worldwide. ${ }^{202}$ Furthermore, a pilot program operated in 2018 by a joint taskforce between the DHS and the U.S. Customs and Border Protection (CBP) shows the advantages of blockchain in addressing cross-border activities similar to supply chains that involve multiple governments and private partners. ${ }^{203} \mathrm{CBP}$ is inspecting over 80,000 shipping containers and $\$ 6$ billion worth of imported goods on a daily basis. ${ }^{204}$ Although CBP has a digital platform, called the Automated Commercial Environment, to process the reporting of import and export and the government approval thereof, the industry still uses numerous redundant paper forms. ${ }^{205}$ The pilot program revealed that a blockchain platform can help replacing the existing paper-based process and, with intensive planning for standardization, a blockchain adopted by CBP may interoperable with other blockchains used by multiple private parties and trading partners. ${ }^{206}$

Thus, despite all its complexity and challenges, blockchain's potential as a decentralized form of record-keeping is almost without limit, even in the public sector. From a bird-eyes view, many examples in the public sector in Part II.C.2. are related to regulatory compliance and reporting from the perspective of citizens, such as FDA regulations, election law, immigration

${ }^{201} \mathrm{Id}$.

202 Id. at 28.

203 Svetlana Angert, Blockchain Technology Implementation in the U.S. Customs Environment (Sep. 2019) (unpublished M.A. thesis, Naval Postgraduate School), available at https://www.hsdl.org/?view\&did=831027.

${ }^{204} \mathrm{Id}$. at 21.

${ }^{205}$ Id.

${ }^{206} \mathrm{Id}$. at 35. 
law, labor law, military law, and customs. Furthermore, many examples in the private sector in Part II.A.1., such as banking, financial services, personal record management, and supply chains, inevitably invite the regulatory compliance aspect. Thus, the public sector relating to compliance and reporting is a good fit to incorporate blockchain. Tax is no exception. By benchmarking the examples discussed in this Part, Part II offers a framework that evaluates whether and what types of taxes are recommended to incorporate blockchain technology as well as explores the possible blockchain architecture that is available for tax administration.

\section{BLOCKCHAIN ARCHITECTURE FOR TAX ADMINISTRATION}

\section{A. Feasibility}

A couple of government-sponsored reports on applying blockchain to governments have been released since 2017, but none of these reports seriously discusses tax as an area that could benefit from the application of blockchain. ${ }^{207}$ However, blockchain technology has already been applied to many areas, such as cash flow and property record-keeping, that are closely integrated with the tax compliance system. Then, is it not worth exploring blockchain initiatives for tax administration? This Part delves into this question.

Existing studies analyzing whether blockchain is recommended for a specific area within the public sector provide four criteria in common: data redundancy, information transparency, data immutability, and a consensus mechanism. ${ }^{208}$ If a sector requires at least three of the four factors, then that public sector is recommended to incorporate blockchain. If a sector requires one or two factors, blockchain might work, but it is likely that simpler or cheaper ways are available instead of blockchain.

Tax administration requires at least three of the four factors outlined above: data redundancy, information transparency, and data immutability. Sometimes tax administration requires a consensus mechanism as well. Tax administration starts with an information asymmetry between taxpayers and tax authorities. To resolve any asymmetry of information and achieve transparency, tax administration requires significant compliance and administration efforts and often data redundancy. For example, the information of a taxpayer's income must be obtained by both the federal government and state local authorities. With payroll taxation, the amount of wage is reported and shared with various government agencies and companies. Various institutions, such as insurance companies and the Social

\footnotetext{
207 See e.g., DATA FOUNDATION, supra note 192; DHS, supra note 10.

${ }^{208}$ Id. at 5.
} 
Security Administration, also collect the same information to process wage income amounts. Thus, the systems impose significant burdens on the intermediaries (i.e., employers), and yet remain far from efficient, because each government agency and institution involved holds their own register, in effect duplicating data held by other institutions. Thus, the supporting system for managing tax information requires constant improvement for efficiency. Furthermore, tax information so achieved must be properly used, stored securely, and protected from any undue disclosure to unrelated parties or the public.

Hence, it is worth examining the idea of integrating blockchain into existing tax administrations. Based on the four criteria noted above, this Article proposes a framework to help categorize areas of taxation that can be benefited from blockchain as follows: 1) reporting obligations of the same information to multiple tax authorities and agencies (e.g., payroll taxation, transfer pricing), 2) third-party reporting obligations (e.g., withholding tax), 3) transaction taxes (e.g., value added tax, customs), and 4) information sharing among tax authorities (e.g., among federal, state, and local governments, among multiple countries). Table 2 summarizes the promising categories and examples in both domestic and international tax, some of which will be illustrated in Subparts C and D.

TABle 2. TAX CATEgORIES AND EXAMPles FOR INCORPORATING BLOCKCHAIN

\begin{tabular}{|c|c|c|}
\hline Category & Domestic tax & International tax \\
\hline $\begin{array}{l}\text { Reporting obligations of } \\
\text { the same information to } \\
\text { multiple tax authorities } \\
\text { and agencies }\end{array}$ & - Payroll tax & $\begin{array}{l}\text { Transfer pricing and } \\
\text { country-by-country } \\
\text { report }\end{array}$ \\
\hline $\begin{array}{l}\text { Third-party reporting } \\
\text { obligations }\end{array}$ & - Withholding tax & $\begin{array}{l}\text { - Withholding tax } \\
\text { - } \text { DAC6 }\end{array}$ \\
\hline Transaction tax & $\begin{array}{l}\text { - Value-added tax } \\
\text { - Sales tax } \\
\text { - Tax on property } \\
\text { transaction }\end{array}$ & - Customs, tariffs \\
\hline $\begin{array}{l}\text { Information sharing } \\
\text { among tax authorities }\end{array}$ & $\begin{array}{l}\text { Among federal, state, and } \\
\text { local governments: } \\
\text { - State Audit Report } \\
\text { Program (SARP) } \\
\text { - State Reverse File } \\
\text { Match Initiative } \\
\text { (SRFMI) } \\
\text { - Municipal Agency } \\
\text { Partnering Program }\end{array}$ & $\begin{array}{l}\text { Among multiple countries } \\
\text { - Bilateral Tax } \\
\text { Information Exchange } \\
\text { Agreement (TIEA) } \\
\text { - Multilateral Tax } \\
\text { Information Exchange } \\
\text { Agreement } \\
\text { Automatic Exchange of } \\
\text { Information }\end{array}$ \\
\hline
\end{tabular}


On the other hand, certain individual income taxes, including selfemployment tax and tax on business income, would not be an ideal situation to incorporate blockchain technology. Part III.C. discusses such limitations and why.

Parts C and D illustrates some of the areas that are recommended in Table 2. As a preview, blockchain can benefit both tax authorities and taxpayers in those areas. Tax authorities can have a better tax data management system that is more efficient and transparent and can process data in real time. In addition to the classic efficiency gain of blockchain that resolves information asymmetry between tax authorities and taxpayers, blockchain can build a more democratic system among tax authorities because it can be decentralized and distributed among federal, state, and localities, all of whom would participate in the blockchain network on the equal footing.

Taxpayers can also benefit from the transparent and efficient blockchain system. They do not have to report their tax information separately to federal and state tax authorities as well as multiple agencies, because blockchain can eliminate the need for redundant data entry. Also, with proper design, their tax information can be more securely protected and shared only among the regulators and institutions that have permission to access such data. The next Subpart envisions the desirable blockchain architecture for tax administration.

\section{B. Recommending a Private Consortium Blockchain}

To determine which type of blockchain is recommended for tax administration, it is helpful to review the pros and cons of various types of blockchains discussed in Part I.B.

In theory, all versions of blockchain are decentralized peer-to-peer networks which utilize some form of a consensus model to verify transactions. Public or permissionless blockchains are typically what first come to mind when one thinks of blockchain. ${ }^{209}$ In public blockchains, anyone can read, send transactions, and participate in the consensus process. The openness of the system prevents one entity from possessing a majority control over the network. ${ }^{210}$ However, the process is very costly because the large number of nodes that are required to verify the transactions consume substantial computation power. ${ }^{211}$ Furthermore, a key aspect of public blockchains is that anyone can access them, unless the public blockchains are permissioned. Thus, it is not likely to recommend public blockchains for tax

${ }^{209}$ Shaverdian, supra note 67, at 1267 (noting how permissionless blockchains are the "most well-known blockchain network.").

${ }^{210}$ Shaverdian, supra note 67, at 1267.

${ }^{211}$ Id. 
administration where taxpayers privacy must be protected.

If having some central control of the blockchain is important, as is expected in tax administration by tax authorities, then a private permissioned blockchain is a better option than a public one. In fully private blockchains, a single organization maintains control over the entire system. ${ }^{212}$ Unlike with public blockchains, not anyone can participate in the network. Users must be invited into the blockchain by either the starter of the network or by a set of rules that were put in place when the network was created. ${ }^{213}$ Private blockchains are always permissioned blockchains, so that the restrictions on access to certain information can be placed on private blockchains. ${ }^{214}$ Also, private blockchains are much more efficient and cost-effective because not as many users are required to validate the transactions. ${ }^{215}$

Hence, private permissioned blockchains, as opposed to public blockchains, would be a recommended design for a blockchain in tax administration where the goal is to let the public or other agencies view certain information while keeping confidential information private. Under this scenario, the central tax authority, such as the IRS, could control the blockchain and modify it as needed. Private blockchains would also be helpful for the tax sector for two reasons. First, tax administration can improve transparency and protect tax privacy at the same time. A private permissioned blockchain can allow the public to see certain statistical tax information, while still limiting the visibility of taxpayers' sensitive information. Therefore, it is possible that the public can use this new public information from the blockchain to judge the effectiveness of the IRS's tax administration, while also limiting sensitive information like names, addresses, and social security numbers from being disclosed. Second, that same blockchain can also improve efficiency beyond tax administration. Private blockchains could be used to disclose mandated information to other government agencies, while at the same time systematically keep confidential information confidential.

Yet, private blockchains can raise governance issues. The blockchain's distributed network system is one of the most important advantages that blockchain can offer, as opposed to central data management, but private blockchains may retreat to another form of central data management system. ${ }^{216}$ In a rare case, it might be possible that the blockchain

${ }^{212}$ Vitalik Buterin, On Public and Private Blockchains, ETHEREum (Aug. 6, 2015), https://ethereum.github.io/blog/2015/08/07/on-public-and-private-blockchains/.

${ }^{213}$ Praveen Jayachandran, The Differences Between Public and Private Blockchain, IBM (May 31, 2017), https://www.ibm.com/blogs/blockchain/2017/05/the-differencebetween-public-and-private-blockchain/.

${ }^{214}$ Shaverdian, supra note 67, at 1268.

${ }^{215} \mathrm{Id}$.

${ }^{216}$ This so-called Vili's governance paradox will be discussed in depth infra Part III.D. 
administrator could act maliciously and cause problems for the entire blockchain, such as excluding certain users, rewriting block history, or deleting resources. $^{217}$

However, this governance problem may be mitigated if the private blockchain is also a consortium, rather than a plain-vanilla private blockchain (distinction discussed in Part I.B.3). Consortium blockchains have all of the benefits of private blockchains while also retaining a "partiallydecentralized" aspect to its operation. ${ }^{218}$ One organization or person does not have all of the control, and thus the blockchain is programmed to ensure that there is consensus amongst participants to take action. Consortium blockchains are also permissioned blockchains that require users to join only by invitation. The blockchain network can be set up so that only a set number of nodes are required to verify a block, rather than requiring all nodes to verify. Consortium blockchains are best suited for participants who want to work together but do not completely trust each other and want to keep some information private. ${ }^{219}$

Therefore, a private consortium blockchain seems the logical type of blockchain solution for tax administration. Consider again the areas that could benefit from blockchain in Table 2. Mostly, the areas involve multiple tax authorities and parties who contribute tax data, which makes a consortium blockchain a promising architecture for tax administration.

A private consortium blockchain can resolve information asymmetry not only between tax authorities and taxpayers, but also among tax authorities and other agencies in the private sector. In particular, consortium blockchains are likely the most helpful for sharing information among tax authorities in interstate and international tax. For example, a consortium blockchain could be set up to only allow certain states or countries to participate, and also allow additional states or countries to join based on consensus by the participating members. Under such circumstances, a traditional plain-vanilla private blockchain would not work because it is unlikely that all countries could decide which country should have all of the control. With this consortium blockchain, exchange of tax information can be executed more efficiently and transparently. Importantly, this process does not need a central authority, making the system democratic among the members. Also, the exchange system could be more secure. Tax information could be exchanged among pre-selected members and further verified without other participating states or countries knowing the content of the information. This allows the relevant tax information to be kept confidential within the entire network while

217 YAGA ET AL., supra note 1, at 49.

${ }^{218} I d$.

${ }^{219}$ What Are Consortium Blockchains?, INFINITY BLOCKCHAIN LABS (Jan. 16, 2018), https://www.blockchainlabs.asia/news/what-are-consortium-blockchains/. 
allowing only pre-selected members chosen by smart contracts to have access to the content.

To illustrate, suppose that the federal government and fifty states create a blockchain network for tax information sharing. If only the federal government and the state of California need to access tax information of a Californian resident taxpayer without sharing such data with other states, such as New York, the consortium blockchain could make that possible while all states, including New York, participate in verifying the accuracy of taxpayer information without knowing the content of the information.

In international tax, suppose that G20 countries create a consortium blockchain network for tax information sharing. If only the United States and the United Kingdom need to access tax information of their dual residents without sharing such data with other countries, such as China, consortium blockchain could make this possible. This feature of blockchain is particularly beneficial for international tax which inherently lacks central authorities that could guarantee the trust in a multilateral cooperation. Parts II.C.3. and D.2. elaborate these domestic and international opportunities.

In sum, a private consortium blockchain is likely the most useful form of blockchain for tax purposes. With the general features of blockchain, a tax blockchain can enhance the efficiency and transparency of the tax admin system by eliminating redundant reporting and data management. With the features of private and permissioned blockchain, participants can limit what information is seen by the various users and the public. This advances the security of the tax administation system and taxpayer privacy. Furthermore, the features of consortium blockchain, as opposed to a plain-vanilla private blockchain, can improve transparency and efficiency among multiple tax authorities, agencies, and private parties who contribute tax data. Consortium blockchains offer the best tools to share information among participants. At the same time, the network would be partially decentralized, so that a consortium blockchain can overcome the potential drawbacks of a plainvanilla blockchain.

Building upon the general architectural recommendation, Subparts $\mathrm{C}$ and D illustrates specific areas of tax administration in domestic and international tax that might benefit from incorporating a blockchain system.

\section{Examples in Domestic Tax}

\section{Payroll Taxes and Beyond}

Payroll taxes generally include taxes for social insurance and hospital insurance, commonly referred to as "Social Security" and "Medicare," that, separately and collectively, are taxes under the Federal Insurance 
Contributions Act (FICA). ${ }^{220}$ In addition, federal, state, and local taxes are also withheld. Although the ultimate tax obligation is shared between employers and employees, employers have the responsibility to withhold the employee's share from their wages and deposit such amounts. ${ }^{221}$ Employers also withhold the employee's federal, state, and local income taxes from the employee's paycheck and pay it to the IRS on behalf of the employee. ${ }^{222}$ Because the FICA taxes and withholding taxes operate in the same payroll system, where the tax base is the employee's income and employers are acting as a withholding agent of the employee, payroll taxes in this Article refer to all taxes withheld from the wages and salaries for simplicity.

If blockchain was incorporated into tax administration, the payroll tax system would be the frontrunner. There are many government agencies and financial institutions involved in the payroll tax, and each one collects the same data and holds their own register centrally. ${ }^{223}$ This duplicates data and overlaps compliance efforts, making it an ideal setting to consider blockchain to improve the systemic flaws. ${ }^{224}$ Furthermore, the fact that the payroll tax system is already digitalized in most developed countries is an additional reason to consider blockchain. ${ }^{225}$

Implementing a blockchain-based system can be done, for example, by embedding smart contracts that fully automate calculating and transferring tax and social security payments from employee salaries to relevant agencies and institutions. ${ }^{226}$ The system can be expanded to include various savings and retirement plans. The process could be done in the following steps: ${ }^{227}$

1. The employer enters the gross amount of compensation into the consortium blockchain system, comprising of the tax authorities, government agencies, financial institutions, and the other necessary parties,

${ }^{220}$ John Olson, What are Payroll Taxes and Who Pays Them?, TAX Foundation (July 25, 2016), https://taxfoundation.org/what-are-payroll-taxes-and-who-pays-them/.

${ }^{221}$ I.R.C. $\S \S 3101-3102,3121$.

222 I.R.C. $\$ 3402(2011)$.

${ }^{223}$ Richard T. Ainsworth \& Ville Viitasaari, Payroll Tax Compliance and Blockchain, 85 TAX NOTE INT'L 1007, 1018 (2017).

${ }^{224} \mathrm{Id}$.

225 Deloitte, Blockchain Technology and Its Potential in TaXes 11 (2017), https://www2.deloitte.com/content/dam/Deloitte/pl/Documents/Reports/pl_Blockchaintechnology-and-its-potential-in-taxes-2017-EN.PDF [hereinafter, DELOITTE, BLOCKCHAIN TAX].

${ }^{226}$ Ainsworth \& Viitasaari, supra note 223, at 1021.

${ }^{227}$ Id. at 1020; see also DeloITTE, BlOCKCHAIN TAX, supra note 225, at 11. 
2. Within the blockchain system, smart contracts match the data of the employee and calculate the correct tax and social security amounts, and

3. The net salary is automatically transferred to the employee's account and the calculated tax is sent to the federal and state treasury, government agencies, and other organizations.

Despite the potential benefits of a faster, less costly, and more efficient process, there are not yet many real-world blockchain applications in the payroll tax system. This is because it requires an extreme level of coordination among the regulatory agencies and other players in the private sector and many are hesitant to try this new technology. ${ }^{228}$ However, a handful of blockchain-based platforms, such as Futurice and Bitwage, offer limited payroll services for processing payrolls domestically and globally. ${ }^{229}$

\section{Value Added Taxes}

Another type of tax where blockchain can provide benefits is the taxation of transactions where multiple parties and intermediaries are involved for collecting and paying the taxes. One example is a value-added tax (VAT). ${ }^{230}$ Instead of taxing a percentage of the entire sales price at the time the goods or services are finally sold to the consumer, as a sales tax normally does in the United States, a VAT imposes a tax on the "value added" to the goods or services during each stage of the supply chain. ${ }^{231}$ Each taxpayer in the supply chain pays VATs on any increase in value that person contributes, which is the difference between the value of an enterprise's sales (outputs) and purchases (inputs). ${ }^{232}$

VATs are considered administratively superior to sales taxes. ${ }^{233}$

228 Ainsworth \& Viitasaari, supra note 223, at 1018.

${ }^{229} \mathrm{Id}$. at 1020.

${ }^{230}$ To be precise, a VAT is a consumption tax, rather than a transaction tax imposed on a particular transaction. A VAT is placed on goods and services whenever value is added at each stage of the supply chain, from production to the point of sale, and each stage involves a transaction and conveyance. In this context, this Article uses VATs to demonstrate tax administrative problems in transactions.

${ }^{231}$ William G. Gale \& Benjamin H. Harris, Proposal 10: Creating an American ValueAdded Tax, THE BROOKINGS Inst. (2013), https://www.brookings.edu/wpcontent/uploads/2016/06/THP_15WaysFedBudget_Prop10.pdf.

${ }^{232}$ Id.

${ }^{233}$ Why is the VAT Administratively Superior to a Retail Sales Tax?, TAX POLICY CENTER (May 2020), https://www.taxpolicycenter.org/briefing-book/why-vatadministratively-superior-retail-sales-tax. 
Therefore, scholars and policymakers advocate for the adoption of VATs in the United States. ${ }^{234}$ Nonetheless, the VAT process is complex and burdensome for taxpayers. A taxpayer must issue invoices (including output VAT), collect output VAT, pay their suppliers bill (including input VAT), and ultimately pay the VAT due (output VAT minus input VAT). ${ }^{235}$

Blockchain has the potential to notably reduce the administrative burden of companies subject to VATs by streamlining the process through a decentralized system. ${ }^{236}$ Every transaction implicating the VAT could be conducted and reported in real time, as opposed to having a team of accountants who have to both dig through all the relevant transactions and calculate the VAT. Further, because of smart contracts, all transactions executed on the blockchain would be tamper proof and transparent, reducing the risk of fraud and mistakes.

Being able to view the effects of the VAT in real time, as opposed to only seeing the effects at the time of reporting, also provides immediate insight into a company's finances. ${ }^{237}$ When paying the VAT via a blockchain platform, high-speed money transfers can take place between businesses and the government. Taxpayers can calculate the VAT amount due at the invoice level instead of the tax return level. Room for VAT fraud would be drastically reduced because the same blockchain system for VAT processing could allow multi-dimensional checks and verifications of the transaction's details, including the legal and business issues of the relevant parties.

In many countries, the VAT is the largest contributor to government tax revenues, and thus, tax authorities are eager to find ways to enhance the efficient collection of VATs. ${ }^{238}$ This suggests that governments may be motivated to try a blockchain solution for their current VAT system. Brazil and Hungary require electronic invoices, making real-time reporting available via blockchain. ${ }^{239}$ Poland is working on creating a daily reporting system of VATs. ${ }^{240}$ The European Union (EU) proposed a blockchain

234 See e.g., Reuven Avi-Yonah, Designing a Federal VAT: Summary and Recommendations, 63 TAX L. REV. 285 (2010); see also William G. Gale, Raising Revenue with a Progressive-Value Added Tax, THE BROOKINGS InsT. (2020), https://www.brookings.edu/research/raising-revenue-with-a-progressive-value-added-tax/.

235 This process is even more burdensome for countries that require the VAT to be reported and paid on a monthly (like in most EU countries) or quarterly basis. EU VAT Returns, AVALARA (last visited Jan. 13, 2021), https://www.avalara.com/vatlive/en/eu-vatrules/eu-vat-returns/eu-vat-returns.html.

${ }^{236}$ DELOITTE, BLOCKCHAIN TAX, supra note 225, at 13.

${ }^{237}$ Id.

${ }^{238} I d$. at 12 .

${ }^{239} I d$.

${ }^{240} I d$. 
solution for the VAT to prevent ongoing VAT fraud. ${ }^{241}$ Even in the Middle East, the Gulf Cooperation Council (GCC) appears to have introduced the first real-time blockchain VAT where some commentators believe that the GCC's blockchain VAT system solves many of the potential fraud problems that exists in the EU's system. ${ }^{242}$

\section{Information Sharing among Federal, State and Local Governments}

Bringing blockchain into tax administration can offer a possible solution to the information asymmetry that currently exists among federal, state, and local governments.

Currently, federal, state, and local governments share specific tax information through various programs such as the State Audit Report Program (SARP), the State Reverse File Match Initiative (SRFMI), and the Municipal Agency Partnering Program. ${ }^{243}$ Those information sharing programs had identified an estimated $\$ 6.8$ billion in tax liabilities from 2013 through $2016 .^{244}$

However, the federal and state government do not equally leverage the shared information. The IRS shares tax information, such as audit results, with states and localities via those programs. ${ }^{245}$ While states and localities also share tax information with the IRS, the IRS has not used "[s]tates audit report information effectively due to differences in the [s]tate laws, report formats, inconsistencies in the use of referrals among divisions, and changing priorities." 246 The resulting information gaps have asymmetrical consequences in tax administration. For example, if only a state finds out about additional income, federal tax repercussions rarely occur. On the other hand, if the federal government finds out about additional income, it is only a matter of time before the state or local governments find out also and challenge the taxpayer.

Another problem is caused by the different focus and laws which govern

${ }^{241}$ See generally Richard T. Ainsworth \& Musaad Alwohaibi, A VATCoin Solution to MTIC Fraud: Past Efforts, Present Technology, and the EU's 2017 Proposal, 89 TAX NoTES INT'L 335 (2018) (discussing the EU's proposal to prevent fraud within their VAT system).

${ }^{242}$ Richard T. Ainsworth \& Musaad Alwohaibi, The First Real-Time Blockchain VAT: GCC Solves MTIC Fraud, 86 TAX NOTES INT'L 695, 696 (2017).

243 Local Information Sharing, INTERNAL REVENUE SERVICE, https://www.irs.gov/government-entities/governmental-liaisons/local-information-sharing (last visited Jan. 14, 2021); see also TREASURY INSPECTOR GENERAL FOR TAX Administration (TIGTA), The Internal REVEnue SERVICE CAN More EFfectively AdDRESS NONCOMPliance By BetTer Using AND CONTROlling THE FED/State PROGRAM 2 (2018).

244 Id. at 7 .

245 Id. at 1.

${ }^{246}$ Id. at 5. 
the state and federal programs. ${ }^{247}$ Because the state tax base can deviate from the federal tax base, states may be interested in different types of information than the federal government. ${ }^{248}$ The amount of information shared also varies from state to state and is limited to the information agreed upon in individual federal/state agreements. ${ }^{249}$ This can cause issues in compiling data, and the data does not serve either party.

Implementing a consortium blockchain among the various governments would grant greater possibility to federal, state, and local governments to collaborate on tax compliance. In the blockchain, not only tax audit information but also other tax-related raw information on tax filers could be recorded. All member states can participate in verifying such information, but only relevant agencies would get permission to access such data. Implementing this type of infrastructure would eliminate the delays in communication. The system would also create a more standardized approach in tax compliance, which would contribute to a path toward greater conformity between state and federal authorities, as many scholars have longed for. ${ }^{250}$

\section{Examples in International Tax}

International tax has ideal conditions that could benefit from incorporating blockchain. Inherently, there is no central government or authority to administer various issues of international tax. But international tax has many areas where tracking down the cross-border cashflow or information is essential for tax administration, yet information asymmetry between relevant governments and taxpayers has been severe. To combat offshore tax evasion and achieve transparency in tax information, the international community has developed many policies, such as country-bycountry reporting for transfer pricing and information sharing among the relevant governments. However, the efficacy of those policy tools is far from perfect because of the fundamental lack of trust on the management of tax data between taxpayers and governments and among relevant governments.

\footnotetext{
${ }^{247}$ Id.

${ }^{248}$ For state and federal tax inconformity, see e.g., Ruth Mason, Delegating Up: State Conformity with the Federal Tax Base, 62 DUKE L.J. 1267 (2013); Erin Adle Scharff, Laboratories of Bureaucracy: Administrative Cooperation Between State and Federal Tax Authorities, 68 TAX L. REV. 699 (2015).

249 TIGTA, supra note 243, at 2.

${ }^{250}$ See e.g., DANIEL ShaVIRO, FEDERALiSM IN TAXATION: THE CASE FOR GREATER UNIFORMITY (1993); Mason, supra note 248. Cf. Scharff, supra note 248 (being more sympathetic to federal-state base nonconformity and rather emphasizing to develop cooperative tax compliance and enforcement program between state and federal tax authorities.).
} 
Without a central authority and its oversight, a taxpayer or a government may well hesitate to voluntarily report and share the tax information with other countries.

However, blockchain enables direct, peer-to-peer data management between parties who do not fully trust each other, or who do not trust any central authority to validate information. With a proper design, such as a consortium blockchain, blockchain could systematically restrict access of a particular tax information by certain countries or parties who are not pertinent to that information even if those countries or parties are a member of the consortium. This Subpart delineates two examples to show how blockchain would improve transparency and resolve information asymmetry in international tax.

\section{Transfer Pricing, Country-by-County Reporting}

International transactions within the ambit of multinational enterprises (MNEs), such as a transaction between a parent company in country A and its subsidiary in country B, are called intra-firm transactions or related-party transactions. ${ }^{251}$ In contrast, international transactions among unrelated parties are called arm's length transactions. Intra-firm trade represents a significant portion of global trade, accounting for about half of global trade volume. ${ }^{252}$

The term "transfer pricing" refers to tax policies and rules to regulate the setting of prices on related-party transactions in international tax. ${ }^{253}$ If related parties could decide transfer prices of intra-firm transactions as they wish, they would have strong incentives to allocate profits to an entity in low-tax jurisdictions and losses to an entity in high-tax jurisdictions. Suppose that Apple Inc., the parent company in the United States, pays royalties to its wholly owned Irish subsidiary for the use of intellectual property rights owned by the Irish subsidiary. ${ }^{254}$ If the corporate income tax rate is $35 \%$ in the United States but the royalty payments are taxed at very low rates in Ireland, Apple, Inc. has very strong incentives to balloon the royalty payment amount that is deductible from its income, because the royalty payment can reduce its U.S. tax liability whereas the royalty income of the Irish subsidiary

${ }^{251}$ Rainer Lanz \& Sebastien Miroudot, Intra-Firm Trade: Patterns, Determinants and Policy Implications, OECD 5 (2011), http://dx.doi.org/10.1787/5kg9p39lrwnn-en.

${ }^{252}$ Intra-firm trade represented $46 \%$ of the U.S. imports and $30 \%$ of U.S. exports in 2009, and about half of export across nine OECD countries. Id. at 5, 12.

${ }^{253}$ CHARLES H. GUSTAFSON ET AL., TAXATION OF INTERNATIONAL TRANSACTIONS 710 (4th ed. 2011).

254 This is a stylized fact of Apple case regarding an EU doctrine known as "state aid." See Apple Sales International and Apple Operations Europe v. European Commission, joined cases T-778/16 and T-892/16 (GCEU 2020); Ruth Mason \& Stephen Daly, State Aid: The General Court Decision in Apple, 99 TAX NOTES INT’L 1317 (2020). 
is subject to no, or little if any, tax in Ireland. As a result, Apple as a group can reduce its global tax liability using this technique, often called base erosion and profit shifting.

Transfer pricing rules are designed to combat such practices that distort taxable income allocable to a particular country. ${ }^{255}$ Tax authorities can adjust intragroup transfer prices if such prices differ from what would have been charged by unrelated enterprises dealing at arm's length. ${ }^{256}$

However, transfer pricing rules are different for each country, and thus create a significant compliance burden for both tax authorities and MNE taxpayers. ${ }^{257}$ In order to assess transfer pricing compliance, many countries require taxpayers to provide transfer pricing documentations, such as intrafirm documents and correspondence to define the role of each involved party and comparable data. ${ }^{258}$ Because each tax authorities require different documentations as what they perceive necessary, although many are overlapping, taxpayers are required to submit similar documents to multiple tax authorities, causing redundant data management efforts. This data is stored centrally by each country individually. Thus, there is significant risk that tax authorities cannot timely detect the possible manipulation of transfer pricing documents by taxpayers. ${ }^{259}$

Blockchain offers a clean solution for this problem. ${ }^{260}$ If a blockchain was used, then intra-firm agreements and other transfer pricing documentations would be recorded on the blockchain. Such data is time-stamped and cryptographically secured, reducing the risk of data manipulation. Tax authorities could easily track the flow of transactions and identity of relevant entities in the group. Furthermore, the blockchain could be designed as a consortium among multiple countries, where MNE taxpayers can enter one documentation in the system without redundant reporting. The information on the blockchain would be only visible to the relevant tax authorities that need to have access to certain information.

Moreover, the Organisation for Economic Cooperation and Development (OECD)'s recent efforts to standardize and coordinate the transfer price reporting, called Country-by-Country Reporting ( $\mathrm{CbC}$ Reporting), can bolster the initiatives to consider blockchain in transfer pricing. Instead of filing separate transfer pricing documentations with different countries, $\mathrm{CbC}$ Reporting requires that only the parent company files a country-by-country

${ }^{255}$ GUSTAFSON ET AL., supra note 253, at 710-12.

${ }^{256} \mathrm{Id}$.

257 OECD, Discussion Draft on TRANSFer PRicing DOCUMENTATION AND CBC REPORTING 3 (2014).

${ }^{258} I d$.

${ }^{259}$ DeloitTe, BlockChain TAX, supra note 225, at 12.

${ }^{260} \mathrm{Id}$. 
report along with a master file, while the local country files to its home country. ${ }^{261}$ There is a common template for CbC Reporting, including the breakdown of the "group's revenue, profits, tax, and other attributes by tax jurisdiction," to give tax administrations a global picture of where MNE profits, tax and economic activities are reported. ${ }^{262}$ As of December 2020, over 89 countries had introduced, or taken steps to implement, $\mathrm{CbC}$ Reporting. ${ }^{263}$

Once the parent company provides its $\mathrm{CbC}$ Report to the tax authority in home country, such country is expected to exchange the report with foreign countries where a member of the MNE group is required to pay tax as a tax resident. $^{264}$ The first automatic exchange of $\mathrm{CbC}$ Reports took place in June 2018, and over 2,700 CbC Reports has been bilaterally exchanged as of December 2020. ${ }^{265}$ These information exchanges are carried out via exchange of information programs that the next Subpart recommends as another great area to incorporate blockchain into the system. ${ }^{266}$

\section{Exchange of Information}

The exchange of information regime in international tax is the most recommended area to incorporate blockchain into the system. In parallel with a federal/state tax blockchain consortium, a multinational blockchain consortium is recommended for international exchange of tax information.

In the late 2010s, many scandals about offshore tax evasion, such as the LGT Bank affair ${ }^{267}$ and the UBS scandal, ${ }^{268}$ raised huge concerns in tax

261 OECD, BASE ERosion AND Profit Shifting (BEPS) ACTION 13 COUNTRY-ByCOUNTRY REPORTING: HANDBOOK ON EFFECTIVE IMPLEMENTATION 9 (2017) [hereinafter, OECD, CBC HANDBOOK]. However, the OECD also acknowledges that there may be certain circumstances where a "constituent entity (i.e., an entity within the MNE group)" is required to file the $\mathrm{CbC}$ report directly with its own tax authority. See also OECD, GUIDANCE ON THE IMPLEMENTATION OF COUNTRY-BY-COUNTRY REPORTING: BEPS ACTION 135 (2019) [hereinafter, OECD, CBC GUIDANCE].

262 OECD, СBC HANDBOOK, supra note 261, at 3, 6).

263 OECD, BEPS ACTION 13 COUNTRY-BY-COUNTRY REPORTING, https://www.oecd.org/tax/beps/beps-actions/action13/.

264 OECD, CBC GUIDANCE, supra note 261, at 5.

265 OECD, ACTIVATED EXCHANGE RELATIONSHIPS FOR COUNTRY-BY-COUNTRY REPORTING, https://www.oecd.org/tax/beps/country-by-country-exchange-relationships.htm (last visited Jan. 14, 2021).

${ }^{266} I d$.

${ }^{267}$ See Lynnley Browning, Banking Scandal Unfolds Like a Thriller, N.Y. TIMES (Aug. 14, 2008), https://www.nytimes.com/2008/08/15/business/worldbusiness/15kieber.html (a former employee of LGT Bank group stole customer data and provided it to the EU and the IRS under a new whistleblower program).

${ }^{268}$ In 2007, Bradley Birkenfeld exposed that UBS advised the U.S. taxpayers to establish 
administration. The rampant offshore evasion was possible because tax information relevant to multiple countries, such as a U.S. citizen's Swiss bank account information, was not shared among relevant tax authorities. This information asymmetry triggered a global discussion to enhance the transparency of international financial and tax data, including bolstering the exchange of information. ${ }^{269}$

An important development was the initiative for automatic exchange of information. ${ }^{270}$ Traditionally, tax information had been exchanged between two countries under the bilateral tax treaty, and the exchange occurred upon request. ${ }^{271}$ However, tax authorities wanted to make the system more robust and proposed the multilateral automatic exchange of information on an annual basis. In 2014, G20 endorsed the automatic exchange of information as the "new single global standard," 272 and the OECD released the Common Reporting Standard (CRS) to standardize the automatic exchange of information process. ${ }^{273}$ As of December 2020, there are over 4,400 bilateral exchange relationships activated with respect to more than 100 jurisdictions committed to the CRS. ${ }^{274}$

Despite such efforts, the system is not yet perfectly efficient and secure. The framework for the automatic exchange of information is based on two multilateral instruments - Convention on Mutual Administrative Assistance in Tax Matters and the CRS Multilateral Competent Authority Agreement. ${ }^{275}$ However, countries have to exchange information bilaterally even if both the sending and receiving parties are members of the multilateral instruments, because there is no central administrator who can collect the information from the whole group and distribute the information only to relevant

foreign shell entities, which then opened offshore accounts at the UBS based on the position that those accounts need not be taxed nor be disclosed to the IRS. Joshua D. Blank \& Ruth Mason, United States National Report on Exchange of Information 2-3 (N.Y.U. Law \& Econ. Research Paper Series, Working Paper No. 14-22, 2014); Itai Grinberg, The Battle over Taxing Offshore Accounts, 60 UCLA L. REV. 304, 325-26 (2012).

${ }^{269}$ Young Ran (Christine) Kim, Engineering Pass-Throughs in International Tax, 56 SAN DIEGO L. REV. 707, 763 (2019).

270 See generally Global Forum on Transparency and Exchange of Information for Tax Purposes, OECD, http://www.oecd.org/tax/transparency/.

${ }^{271} \mathrm{Kim}$, supra note 269 , at 764.

272 OECD Delivers New Single Global Standard on Automatic Information, OECD (Feb. 13, 2014), https://www.oecd.org/tax/oecd-delivers-new-single-global-standard-onautomatic-exchange-of-information.htm.

273 See OECD, Standard For Automatic Exchange Of Financial Account INFORMATION IN TAX MATTERS (2014), https://www.oecd.org/ctp/exchange-of-taxinformation/standard-for-automatic-exchange-of-financial-account-information-for-taxmatters-9789264216525-en.htm.

274 OECD, INTERNATIONAL FRAMEWORK FOR THE CRS, https://www.oecd.org/tax/automatic-exchange/international-framework-for-the-crs/.

${ }^{275} \mathrm{Id}$. 
parties. ${ }^{276}$ This still results in a redundant data management setting. Furthermore, the United States has not committed to any multilateral instrument primarily because of the privacy concerns, and rather built its own automatic exchange of information network pursuant to the Foreign Account Tax Compliance Act. ${ }^{277}$ This shows how international system becomes ineffective when there is no central authority and countries do not fully trust each other.

A consortium blockchain can overcome the systemic defect of international tax administration. A consortium blockchain for the exchange of information can be set up to only allow certain countries to participate, and also allow additional countries to join based on consensus by the participating countries. Smart contracts embedded in the blockchain enable tax information to be shared only among pre-selected countries and be further verified without other participating countries knowing the content of the information. This allows the tax information in the blockchain to be kept confidential while allowing only the pre-selected countries involved in the information sharing to have access to the content. All exchanges would occur automatically through smart contracts without having additional steps necessary to execute bilateral exchanges. Indeed, the exchange of information in international tax is the classic efficiency environment that can harvest the most benefits from blockchain.

\section{NORMATIVE CONSIDERATIONS FOR BLOCKCHAIN IN TAX}

Part II demonstrated that there are promising applications where blockchain can improve tax administration by fixing information asymmetry among taxpayers, tax authorities, and beyond. The use of blockchain can enhance the efficiency and transparency of tax administration and strengthen taxpayer privacy and the confidentiality of their tax information with a proper design, such as a consortium blockchain. Part III proposes the normative considerations of the blockchain initiatives for tax administration, such as timeline, standardization, integration with other systems, limitations, and accompanying legislation for taxpayers' rights and privacy.

\section{A. When is a Good Time to Incorporate Blockchain?}

The appropriate timeframe for blockchain implementation in tax administration depends on the timing of widespread use of distributed ledger

276 OECD, ACTIVATED EXChANGE RElAtionship FOR CRS INFORMATION, https://www.oecd.org/tax/automatic-exchange/international-framework-for-thecrs/exchange-relationships/.

${ }^{277} \mathrm{Kim}$, supra note 269 , at 766. 
technology in various sectors of society.

There has been skepticism on whether blockchain will become readily available as a technology, eventually achieving mainstream adoption. ${ }^{278}$ The skeptics argue that blockchain is overhyped and will wither away with time. However, blockchain has already begun to replace existing systems showing that the technology is not going away soon. A recent survey by Deloitte showed that while $50 \%$ of respondents answered that blockchain is overhyped, $88 \%$ of respondents believe that blockchain will eventually achieve mainstream adoption. ${ }^{279}$ This positive belief in regard to blockchain is increasing with time, from $84 \%$ in 2018 and $86 \%$ in $2019 .{ }^{280}$ Furthermore, about $40 \%$ of respondents reported that they have already adopted blockchain into their businesses in 2020, which is a substantial increase from $23 \%$ in $2019 .^{281}$

Marco Iansiti and Karim Lakhani of Harvard Business School offered a useful tool to assess the extent of the development of blockchain technology and the anticipated path about how the technology will be applied in the real world, ${ }^{282}$ which Richard Ainsworth further developed. ${ }^{283}$ Iansiti and Lakhani provided four phases showing the process of societies adoption of new technologies, such as blockchain, that could change the fundamentals of society. Chart 1 describes the four phases based on a two-by-two matrix with two axes - i) the degree of novelty, and ii) the amount of complexity and coordination required to apply such technologies to the real world.

\section{Chart 1. The Four Phases of Technology DeVelopment ${ }^{284}$}

278 See e.g., Shaverdian, supra note 67, at 1286.

279 DeloitTE, SuRVEY, supra note 20, at 5. The survey polled about 1,500 senior executive and practitioners in 14 countries and regions, who have broad understanding of blockchain.

${ }^{280} \mathrm{Id}$.

281 Id. at 7.

${ }^{282}$ Iansiti \& Lakhani, supra note 25.

283 Ainsworth \& Viitasaari, supra note 223, at 1008-18.

${ }^{284}$ Id. at 1017; Iansiti \& Lakhani, supra note 25, at 7. This chart is recreated and developed by the author. 


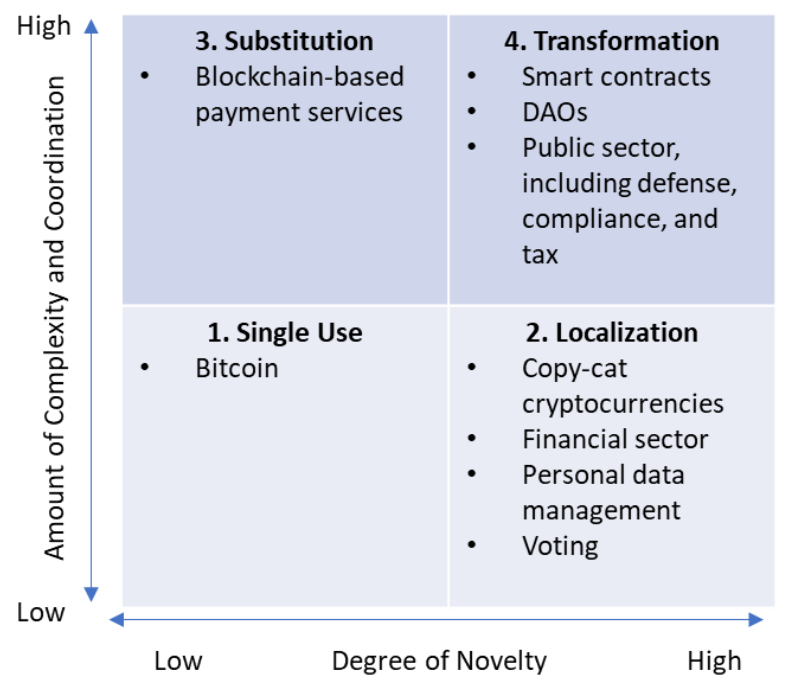

The first phase is a single-use case, where i) an emerging technology has a low-level novelty and ii) the use of technology is not complex and does not require a lot of coordination with other infrastructure. ${ }^{285}$ Bitcoin is an example of single use case in blockchain.

The second phase is localized-use cases. After a single use case, the emerging technology develops to the next level of novelty, but the level of complexity and coordination for the use and application remain in a low level. The "proliferation of copy-cat cryptocurrencies" is a good example of localization of blockchain. ${ }^{286}$ In addition, many applications in Part I.C., such as banking, post-trading processing, managing personal records relating to health or financial data, and voter fraud prevention, are mainly related to this phase.

The third and fourth phases push the level of technology development to a higher level of complexity and coordination. The third phase, substitution, requires a low-level of novelty, whereas the fourth phase requires a high-level of novelty. Blockchain applications that replace traditional business, such as payment services explained in Part I.C.1.c., are in the third phase.

The fourth phase of transformation is the most advanced because it requires both a high level of novelty and a high level of complexity and coordination. In this phase, the technology "could change the very nature of economic, social, and political system" and its application requires significant amount of coordination with the existing institutions. Commentators consider that self-executing smart contracts and DAOs are in this phase. Furthermore, most blockchain applications in the public sector would be in the fourth phase. A defense system deploying military supplies

\footnotetext{
285 Ainsworth \& Viitasaari, supra note 223, at 1009-12.

${ }^{286}$ Id. at 1012 .
} 
via 3D printers, a regulatory compliance system, or a tax system incorporating blockchain would be examples of transformation as these systems would involve coordinating the activity of many actors and require institutional agreement on standards and processes.

Currently, the blockchain technology seems to be in the second and third phases and started entering the fourth phase. The examples of the second and third phases are already in place, and many businesses study to deploy smart contracts in their business model. Part I.C.2 showed the increasing number of projects in the public sector to incorporate blockchain. Thus, the blockchain application in the tax sector that this Article explores is expected to occur in the fourth phase. It is difficult to predict how soon the fourth phase will be prevalent. But considering that the fourth phase has already started, the application of blockchain in tax administration might occur sooner than many expect.

\section{B. Standardization and Integration}

The recommended areas of taxation to incorporate blockchain in Part II.A. are heavily intertwined with other sectors, such as financial institutions, other regulatory agencies, and foreign governments. Because other actors may have their own blockchain networks, a tax blockchain network would need to connect with them seamlessly. Platform fragmentation is not desirable. The more sectors that ultimately adopt blockchain networks, the more standardization will be required. This obvious statement, however, would require significant effort across the board.

A commentator considers that blockchain technology has not yet matured enough to integrate a blockchain network with existing systems or other blockchain networks. ${ }^{287}$ However, there are strong need for standardization of blockchain technology to improve interoperability, adaptability, and capability of integration. Blockchain may grow exponentially with standardization because standardization will eliminate some of the hurdles that prevent the adoption of blockchain. ${ }^{288}$ According to an interview with the author, a tax expert in a leading blockchain network believes that standardization of blockchain technology is the key for the success and advancement to the next phase of blockchain application in both the private and public sector. ${ }^{289}$

As a preliminary issue, there are discussions on whether standardization

\footnotetext{
${ }^{287}$ BASHIR, supra note 26, at 585.

${ }^{288} \mathrm{Id}$. at 586.

${ }^{289}$ Interview with Liz Chien, VP of Tax at Ripple Labs, in San Francisco, Cal. (Oct. 4,
} 2019). 
may harm innovation and competition. ${ }^{290}$ Generally, standardization promotes competition. ${ }^{291}$ Standard setting promotes interoperability of different technologies providing similar services by allowing manufacturers to innovate and compete to provide products conforming to the same requirements. ${ }^{292}$ However, if the standards are proprietary, controlled by big financial and tech firms, and inaccessible to competitors, then standardization could harm innovation and competition. ${ }^{293}$

With those concerns in mind, many countries, such as the United States and the EU, develop standards through standards development organizations (SDOs), rather than by letting a market leader lead the standard-setting processes. ${ }^{294}$ These SDOs develop standards through the work of technical committees, consisting of volunteering experts in the industry. ${ }^{295}$ However, the relationship between SDOs and the governments are different in the EU and the United States. In the EU, the government can play a key role in planning and initiating standardization at the SDOs level, so that the resulting standardization system by SDOs is coordinated with, and directly regulated by, the governments. ${ }^{296}$

On the other hand, the United States takes a more indirect and informal approach to collaborate with SDOs. ${ }^{297}$ The National Technology Transfer and Advancement Act requires government agencies only to use private standards that have been developed through a voluntary consensus process, limiting the role of government agencies in the process to be indirect. ${ }^{298}$ In short, in the U.S., there is no governmental entity that has authority to command an SDO to develop or maintain a particular standard. Thus, for the U.S. to implement a standardization for blockchain technology, including those applicable to tax administration, the governmental entities will be required to work with SDOs on the same footing as those in the private sector

290 Tim S. Simcoe \& Allan L. Shampine, Economics of Patents and Standardization: Network Effects, Hold-Up, Hold-out, Stacking, in THE CAMBRIDGE HANDBOOK OF Technical Standardization Law, Competition, Antitrust, And Patents 104-18 (Jorge L. Contreras ed. 2017).

${ }^{291}$ Id. at $102-104$.

${ }^{292}$ Samuel N. Weinstein, Blockchain Neutrality, GA. L. REV. 34 (forthcoming).

${ }^{293}$ Id. at 50-51.

${ }^{294}$ Id. at 50; Simcoe \& Shampine, supra note 290, at 98; Emily S. Bremer, Government Use of Standards in the United States and Abroad, in THE CAMBRIDGE HANDBOOK OF TECHNiCAL Standardization LAw, Further InTERSECTIONS OF PUbliC AND PRIVATE LAW 29 (Jorge L. Contreras ed. 2019).

${ }^{295} \mathrm{Id}$.

296 Id. at $37-40$.

${ }^{297}$ Id. at 32-35.

${ }^{298}$ National Technology Transfer and Advancement Act 1996, sec. 12(d). Bremer, supra note 294 , at 32 . 
when developing the appropriate standard. ${ }^{299}$ Once developed, the government can legally enforce the adopted private standards by incorporating them in federal regulations. ${ }^{300}$

Interestingly, there are two different attempts to address standardization in blockchain. First, on the international level, the ISO, an independent, nongovernmental international organization with a membership of 165 national standards bodies, established a technical committee, called ISO/TC 307, to study the scope of standardization of blockchain technology. ${ }^{301}$ Second, open source blockchain platforms, such as R3 and Hyperledger, are contributing to the standardization of the blockchain technology by sharing the ideas and codes with other participants in consortia. ${ }^{302}$ Consortia built upon R3 or Hyperledger have at least dozens, if not hundreds, of members who adopt the same blockchain architecture, and they are connected with other blockchain networks, creating a blockchain ecosystem. ${ }^{303}$ This in a way results in standardization.

These efforts demonstrate the need for standardization of blockchain technology. As to the blockchain for tax administration, standardization is essential, because a tax blockchain network needs to be connected with other sectors, such as financial networks and other regulatory networks, to be successful. Thus, policymakers should consider standardization and interchangeable modules for a successful tax blockchain network. It is worth emphasizing that consortium-based blockchains, which are recommended by this Article, are a good way to achieve standardization. Furthermore, policymakers and regulators should diligently participate in the standardsetting process alongside SDOs to make sure that the standardization is appropriate and to allow for the standardization to be incorporated in regulations in a later stage.

\section{At the Intersection Between Offline and Digital: Limitations of Reducing Tax Gap}

Although blockchain may improve tax administration, it is important to understand its limitations. Blockchain is most useful when data are high

299 Id. at 30.

${ }^{300} \mathrm{Id}$. at 33.

301 See Technical Committees ISO/TC 307, Blockchain and distributed ledger technologies, INTERNATIONAL ORGANIZATION FOR STANDARDIZATION (ISO), https://www.iso.org/committee/6266604.html (last visited Jan. 15, 2021); BASHIR, supra note 26 , at 585 .

${ }^{302}$ Id.

${ }^{303}$ Id. For the R3 Blockchain Ecosystem, see https://www.r3.com/ecosystem/ (last visited Jan. 15, 2021); see also HYPERLEDGER CONSORTIA, https://www.hyperledger.org/about/join (last visited Jan. 15, 2021). 
quality and already digitalized. At the intersection between the old-school physical data and its digital representation, the effectiveness of the blockchain technology has to rely on humans who can correctly and honestly bridge the "last mile" between the two forms of the same data. ${ }^{304}$ If humans in charge of translating physical data to digital or entering digital data make a mistake or manipulate the data input, there is nothing blockchain can do.

Such constraint due to the human errors is not limited to blockchain technology. Rather, it is a common problem in most data management system, regardless of physical or digital data. Humans who are in charge of entering data in a ledger can manipulate the data even if the ledger is physical, so is the case if the ledger is digital, such as blockchain. The key point here is that blockchain or distributed ledger technology has the same set of problems as other data management systems.

Thus, although blockchain would be a next phase of digital information management system, the benefits of its application are limited to an incremental improvement of the existing system of data management. To illustrate, let us examine whether blockchain may resolve the three prominent tax noncompliance since the twentieth century, according to James Alms el at.: "(1) the failure to report cash payments and receipts, (2) the use of sophisticated tax shelters to manufacture noneconomic losses, and (3) the establishment of hidden offshore account." ${ }^{305}$ Blockchain is promising to resolve the third problem by enhancing the transparency of cross-border cashflow, as shown in Part II.D. However, blockchain is not likely to enhance tax compliance of the first and second categories. The second category about tax shelters may be improved by other emerging technology, such as artificial intelligence and machine learning. But blockchain is not likely to resolve the first problem about cash business, because blockchain itself cannot improve the integrity of data input by taxpayers.

This reveals the limitation of blockchain to improve an important issue in tax administration, commonly referred to as the "tax gap." The tax gap is the difference between total taxes owed to the government if taxpayers were fully compliant and taxes actually paid on time. ${ }^{306}$ According to the IRS's recent statistics, the IRS should have collected \$2,683 billion each year between 2011 and 2013, but $\$ 381$ billion each year was not eventually collected, which amount not collected is called the tax gap. ${ }^{307}$ This means that $\$ 1$ out of

\footnotetext{
304 Tucker \& Catalini, supra note 6.

305 James Alm et el., New Technologies and the Evolution of Tax Compliance, 39 VA. Tax Rev. 287, 304 (2020).

${ }^{306} \mathrm{Id}$. at 290 .

${ }^{307}$ To be precise, this amount is net tax gap, as opposed to the gross tax gap of $\$ 441$ billion before the IRS's audit and collection efforts. INTERNAL REVENUE SERVICE
} 
every $\$ 7$ of tax due was not paid.

The three major groups of offenders contributing to the tax gap include: i) non-filers, ii) under-reporters who file their tax returns on time but understate their true tax liability, and iii) under-payers who file their returns but fail to pay in full. The second under-reporters group account for the $80 \%$ of the tax gap, so that most efforts addressing the tax gap focus on this group. ${ }^{308}$

Out of the $\$ 352$ billion underreporting tax gap in 2011-13, underreporting on individual income tax returns alone, including self-employment tax, was $\$ 245$ billion, consisting about $70 \%$ of the underreporting tax gap. ${ }^{309}$ Almost $45 \%$ of the underreported individual income tax is owed on business income, which the IRS has no easy way to verify independently when "taxpayers are intentionally noncompliant and conduct business in cash with poor or nonexistent record keeping." 310 In contrast, only about $11 \%$ of the underreporting gap was attributable to corporate income tax, and $20 \%$ to the employment tax, including payroll tax. ${ }^{311}$

Furthermore, when segmenting the individual income tax underreporting tax gap further by the type of income, individual taxpayers fail to report about $55 \%$ of income from sources for which there is little or no information reporting, such as business income from sole proprietorships. ${ }^{312}$ In contrast, only $5 \%$ of income from easily verified sources subject to substantial information reporting, such as pensions, unemployment compensation, dividends, and interest, goes unreported. ${ }^{313}$ When income is subject to both information reporting and withholding tax, as with wages and salaries, only about $1 \%$ goes unreported. ${ }^{314}$

Unfortunately, the categories where tax gap is not significant, such as taxes on income with the easily verifiable sources, payroll taxes, and corporate income taxes, are where the current recommendations exist to incorporate blockchain. Tax gaps on other types of income, such as individual business income, cannot be reduced by simply introducing blockchain into the tax system as long as the problem is deeply rooted in the failure to report cash payments and receipts.

In short, blockchain is not a silver bullet for tax data management or

Research, Applied analytics \& Statistics, Federal tax Complance Research: Tax Gap Estimates for TaX Years 2011-2013, Publication 1415 (Rev. 9-2019) 1 (2019).

${ }^{308} I d$. at 11 .

${ }^{309} \mathrm{Id}$.

${ }^{310} I d$.

${ }^{311} I d$. at $11,16-17$.

${ }^{312} \mathrm{Id}$. at 14.

${ }^{313} \mathrm{Id}$.

${ }^{314} \mathrm{Id}$. 
resolving the tax gap problem. A more effective solution for those areas with big tax gaps would be to introduce a third-party reporting obligation or withholding tax system. Then, those area would fall under the first or second category of Table 2 in Part II.A. that are recommended to incorporate blockchain.

\section{Vili's Governance Paradox and the Role of Tax Authorities}

A salient and important benefit of blockchains as distributed ledger technology is that "they can eliminate the need for a central authority." 315 However, this is not a correct statement not only for private, permissioned blockchains but also public and permissionless blockchains. Blockchains need code developers and engineers when they are developed and continue to need decision makers for governance issues when operated. These key players serve a "a de-facto central authority" in blockchain governance structure. ${ }^{316}$ Thus, blockchains need to nominate trustworthy administrators who are authorized to alter the ledger. But this contradicts with the decentralized characteristics of blockchains. When blockchain networks embrace such governance structure, it is not entirely accurate to describe it as decentralized. ${ }^{317}$

This governance paradox in blockchain is called "Vili's Paradox," named after Vili Lehdonvirta, who first introduced this concept. ${ }^{318}$ Vili Lehdonvirta, who is an economic sociologist at the Oxford Internet Institute and one of the candidates of the true identity of Satoshi Nakamoto who developed Bitcoin (although Lehdonvirta denied), is the one of the first people who explored the governance issues of blockchains. Kevin Werbach of the Warton School responds to Vili's Paradox by distinguishing the rule-creation stage and ruleenforcement stage. ${ }^{319}$ He explains that Vili's Paradox may uphold in the rulecreation stage of blockchains, but the rule-enforcement stage is still decentralized. ${ }^{320}$ What blockchain has eliminated is the need for a trustworthy third party who can verify the information that would be recorded in the ledger. Thus, Werbach vindicates the possibility of blockchain applications to various systems with different degrees of centralization. ${ }^{321}$

Nonetheless, the Vili's governance paradox raises an important question in creation and operation of blockchains: who should be the legitimate

315 BERRYHILL ET AL., supra note 37, at 30.

${ }^{316} I d$.

317 WERBACH, supra note 107, at 133.

318 Id.; Joshua Davis, The Crypto-Currency, THE NEW YORKER (Oct. 3, 2011), https://www.newyorker.com/magazine/2011/10/10/the-crypto-currency.

${ }^{319}$ WERBACH, supra note 107, at 134.

${ }^{320} \mathrm{Id}$.

${ }^{321} I d$. 
governing entity or administrator of blockchains $?^{322}$ For blockchains in the public sector, it raises additional question: what should be the role of the administrators?

The proposed blockchain networks for tax administration are private consortium networks. This means that individual taxpayers cannot participate in the network as a node. Only tax authorities, other agencies, certain withholding agents, and third-party reporters can participate in the network and serve as a node. This would raise concerns on taxpayer rights and privacy for tax information recorded in the blockchain. A taxpayer may want to exercise the right to be forgotten when the taxpayer dies or when a certain statute of limitation expires. ${ }^{323}$ A taxpayer might want to verify and correct certain tax information about herself. Can the taxpayer exercise any rights to protect her information and tax privacy? Because of its immutability, it may be difficult to exercise the right to be forgotten or right to correct the information once the information is recorded in blockchain. ${ }^{324}$

Two options might be considered. First, blockchain system can nominate trustworthy administrators who are authorized to alter the ledger. ${ }^{325}$ For blockchains for the public sector, government officials will have such authority to control the ledger. ${ }^{326}$ However, this may contradict with the decentralized characteristics. Second, the system may destroy the decryption keys and make the data unreadable, instead of compromising the immutability. ${ }^{327}$ But an administrator may easily restore the decryption keys. Furthermore, it may be a solution for the right to be forgotten, but not for the right to correct the data.

Hence, commentators largely recommend the first option over the second and admit the need for administrators for blockchain operation. ${ }^{328}$ Putting the system in tax administration, tax authorities in the blockchain network can perform that role. ${ }^{329}$ As an administrator, the government must carefully consider taxpayer rights and privacy in the rule-creation stage, such as which information should be recorded in the tax blockchain, resulting the data entered becoming immutable, and which information should not be recorded

322 BERRYHILL ET AL., supra note 37, at 30.

${ }^{323}$ Shaverdian, supra note 67, at 1287.

${ }^{324}$ BERRYHILL ET AL., supra note 37, at 29.

${ }^{325}$ Shaverdian, supra note 67, at 1287.

${ }^{326}$ BERRYHILL ET AL., supra note 37, at 29.

${ }^{327}$ Shaverdian, supra note 67, at 1287.

328 See e.g., BERRYHILL ET AL., supra note 37, at 30.

${ }^{329}$ However, the governance issue may persist if there are multiple government entities are involved in a blockchain network. For example, in a consortium blockchain consisting of multiple tax authorities, such as federal, state, and local tax authorities in domestic tax and multiple countries in international tax, the participants still need to decide which authorities would be in charge of the role of an administrator. 
in the tax blockchain. ${ }^{330}$ This discretion requires the government to build a technical knowledge base to ensure that these decisions are made well.

The blockchain governance issue is not peculiar to tax administration, but is rather a general tension between data immutability in a blockchain and the necessary modification, particularly for private consortium blockchains. Though it is beyond the scope of this Article to propose a concrete solution for who to control the system and how to protect taxpayers' rights and privacy, this is an essential issue to be contemplated when the governments consider incorporating blockchain in tax administration. Next Subpart further discusses the taxpayer privacy in blockchain.

\section{E. Taxpayer Privacy: The Case of Undocumented Taxpayers}

The rules of a blockchain system, especially private or consortium blockchain, enable some safeguards for access to private or confidential information. This may strengthen taxpayer privacy or confidentiality for sensitive data. However, if certain sensitive tax data must be shared with other government agencies under laws and regulations, blockchain itself is far from a cure-all for taxpayer privacy.

Let us examine whether blockchain can improve taxpayer privacy concerns in the case of undocumented taxpayers. There is consensus among scholars that, on average, most undocumented immigrants pay taxes. ${ }^{331}$ In 2017, the Pew Research Center estimated that 8 million undocumented persons are in the U.S. workforce, and of those, 3.4 million (nearly half), paid social security taxes. ${ }^{332}$ The Social Security Administration (SSA) stated that unauthorized workers contributed roughly $\$ 13$ billion in payroll taxes in $2010,{ }^{333}$ but it does not track how many pay income taxes. Nonetheless, that 3.4 million number regarding social security taxes sheds some light. Because social security taxes are most often taken from a person's W-2 salary, it follows that those same undocumented people with W2-based jobs likely also have income taxes withheld from their paychecks.

\footnotetext{
330 BERRYHILL ET AL., supra note 37, at 30.

${ }^{331}$ Francine Lipman, The "Illegal" Tax, 11 ConN. PUB. INT. L.J. 93, 107 (2011); Nneka Obiokoye, Taxation of Undocumented Immigrants: The Uneasy Connection Between Regulating the Undocumented Immigrant and Fostering Illegal Activity, 2 Bus. ENTREPRENEURSHIP \& TAX L. REV. 359, 364-67 (2018); Evan Nolan, Picking Up After the Baby Boomers: Can Immigrants Carry the Load?, 24 GEO. IMMIGR. L.J. 77, 85-86 (2009) (noting between one-half and three quarters of all undocumented workers pay taxes); Andrew Tae-Hyun Kim, Deportation Deadline, 95 WASH. U.L. REV. 531, 557-58 (2017).

${ }^{332}$ These numbers are based on reporting by the SSA. Id.; Octavio Blanco, Why Undocumented Immigrants Pay Taxes, CNN (Apr. 19, 2017), https://money.cnn.com/2017/04/19/news/economy/undocumented-immigranttaxes/index.html.

${ }^{333}$ Id .
} 
However, the undocumented workers also fear filing documentation with any government agency because they do not want to be discovered and ultimately be deported. ${ }^{334}$ Technically, undocumented persons are not allowed to work in the United States, and therefore should not be working in W2-based employment. But many still obtain such jobs. Undocumented workers often use a citizen's social security number when seeking employment, and therefore, receive $\mathrm{W}-2 \mathrm{~s} .{ }^{335}$ Then, a problem arises when they try to file taxes. A fake social security number will allow undocumented persons to work, but they are unable to use that same social security number to file taxes. ${ }^{336}$ Therefore, those same workers will have to obtain an Individual Taxpayer Identification Number (ITIN) from the IRS if they wish to file a return. ${ }^{337}$ Although it is against the law to use a fake or stolen social security number to gain employment, the IRS has issued formal guidance to ensure undocumented persons that there will not be any consequences from the IRS for using a false social security number to obtain employment, so long as they use their correct ITIN on their tax forms. ${ }^{338}$ This is all in an effort to increase undocumented taxpayers' confidence and ensure them that they can safely file taxes without fear of being deported.

The IRS would like to increase undocumented taxpayer confidence with a promise of confidentiality to encourage them to continue filing returns. However, undocumented persons still fear filing documentation with any government agency. Therefore, a strange phenomenon exists here. Although most undocumented persons, especially those with W-2-producing jobs, do pay taxes, many scholars believe that fear comes into play when undocumented persons are deciding whether or not to file a tax return. ${ }^{339}$ In many instances, undocumented persons fear filing a tax return and ultimately identifying themselves. This means that most undocumented persons are likely paying more taxes than they should. When employers withhold taxes from each employee's paycheck, it is common that the employer withholds more than the taxpayer's actual tax burden, which can be fixed when the

\footnotetext{
${ }^{334}$ See, e.g., Lipman, supra note 331, at 107 (noting how undocumented immigrants fear deportation and therefore many "do not prepare and file tax returns."); Obiokoye, supra note 331, at 383-84; Leo P. Martinez \& Jennifer M. Martinez, The Internal Revenue Code and Latino Realities: A Critical Perspective, 22 U. FlA. J.L. \& PUB. POL'Y 377, 389 (2011); Chrystin Ondersma, Undocumented Debtors, 45 U. MICH. J.L. REFORM 517, 555 (2012).

335 Obiokoye, supra note 331, at 383-84.

336 Blanco, supra note 332.

${ }^{337}$ Id.; Obiokoye, supra note 331, at 367 (describing an ITIN as one assigned to a foreign national or other person who does not qualify for a social security number).

338 Obiokoye, supra note 331 , at 375.

${ }^{339}$ Lipman, supra note 331, at 96, 101 (noting how "billions" of dollars are paid in taxes each year by undocumented immigrants).
} 
employee files tax returns. ${ }^{340}$ However, if undocumented persons do not subsequently file their tax returns out of fear, they lose the money they overpaid throughout the year. Others point out that undocumented persons do tend to file their tax returns at the end of the year, because they believe that doing so will bear positively on their character if they are ever before an immigration judge. ${ }^{341}$ If they do not file a tax return, it can be seen as evading the law and they also run the risk of having a deficit on their taxes, which they never pay if they do not file a return. In any event, it seems that undocumented persons frequently file taxes, but do so with fear of deportation.

The IRS is required by law to keep tax information confidential from the public and all other government entities. ${ }^{342}$ Therefore, in theory, undocumented persons should not fear that they will be discovered by filing tax returns. However, the confidentiality requirement is filled with exceptions. For example, the IRS is obligated to disclose tax return information to law enforcement investigating non-tax crimes and the IRS may also disclose information regarding payroll and income taxes to the SSA. ${ }^{343}$ Furthermore, although the SSA is required to keep that information confidential, the SSA must disclose certain non-tax information it receives from the IRS to the DHS and the USCIS. ${ }^{344}$ This information includes names, addresses, and other sensitive identifying information. ${ }^{345}$ Therefore, even though the IRS does keep taxpayer information confidential, there are many exceptions provided by laws and regulations that expose undocumented immigrants to the risk of deportation.

Blockchain has the potential to ensure that only the permissible information is released to other federal agencies. Hopefully this creates a more transparent process while also keeping some privacy for undocumented immigrants. A tax blockchain could hypothetically be programmed to allow the IRS to only disclose to other agencies the information required by law. Such programming must be accompanied by legislation that increases privacy for undocumented taxpayers and prohibits the IRS from releasing sensitive information to any other agency like the SSA, DHS, or USCIS. Otherwise, a tax blockchain would not make the process more confidential. Even if the IRS were to only release the mandated information to immigration agencies, and all other information was restricted by the blockchain, that

\footnotetext{
${ }^{340}$ Lipman, supra note 331, at 101.

${ }^{341}$ Blanco, supra note 332.

${ }^{342}$ Id.; Obiokoye, supra note 331, at 375.

${ }^{343} I d$. at 376.

${ }^{344}$ Id. at $376-77$ (much disclosure requires specific requests by the DHS or USCIS, however, those agencies can nonetheless obtain that information).

${ }^{345} I d$.
} 
mandated information consists of all of the identifying information that the undocumented person wants to keep confidential. Therefore, accompanying legislation is needed to make the blockchain technology useful to protect undocumented persons, and more generally, taxpayer privacy.

\section{CONCLUSION}

As blockchain technology develops, it will grow beyond the early stages of a single use case and localization into the substitution and transformation phases. Scholars, engineers, and users emphasize blockchain's original technology as distributed, immutable, peer-to-peer ledger for future data management systems. The evolution from public blockchains to private and consortium blockchains also expands the scope of blockchain applications.

Blockchain has shown promising applications in the private sector, such as financial services and supply chains. But this Article focuses more on the blockchain's potential to play a greater role in the public sector, such as property records, public health, and compliance, where data redundancy, information transparency, data immutability, and a consensus mechanism are required. Tax administration is one of promising applications in the public sector, and this Article recommends the adoption of a private consortium blockchain when architecting the system.

Some might see an irony with blockchain, centered on the idea of decentralization, being used in the public sector, such as in tax law, because "blockchain heralds revolutionary decentralized economic order," 346 hoping to depart from the arguably authoritative government oversight as in a George Orwell's novel, Big Brother. However, reality is that the advantages of blockchain, such as transparency, efficiency, data integrity, and security, can also benefit the public sector in tremendous ways. Specifically, the feature of decentralization can improve the tax administration among multiple tax authorities by offering more equitable setting for all stakeholders. In international tax, there are areas where tracking down the cross-border cashflow and information is important, but the information asymmetry has been severe because there is no central government or authority. Similarly, in domestic tax, the information sharing among federal and state and localities has been far from ideal. Blockchain can enable direct, peer-to-peer data management between parties who do not fully trust each other, or who do not trust any central authority to validate information. Thus, blockchain may suggest a new path for improving tax administration regardless of various power dynamics involved.

Blockchain may not be a silver bullet for tax data management because the technology itself faces some implementation issues, including a

\footnotetext{
${ }^{346}$ WERBACH, supra note 107, at 134.
} 
steep trade-off between efficiency and decentralization, explained as Vili's Paradox. However, by using blockchain in the right ways, such as when data is high quality, blockchain can revolutionize society in many ways. Yet, any benefits of blockchain cannot materialize when quality data may never make their way onto the blockchain in the first place.

Finally, tax authorities must carefully perform the role of administrator on the tax blockchain network to protect taxpayer rights and privacy. Blockchain has potentials to enhance tax administration and taxpayer privacy at the same time, as in the case of undocumented taxpayers in filing their taxes. However, to truly be effective, blockchain technology must be accompanied by additional privacy legislation surrounding the release of tax information. 